\title{
The classification and the conjugacy classes of the finite subgroups of the sphere braid groups
}

\author{
DACIBERG L GONÇALVES \\ JOHN GUASCHI
}

\begin{abstract}
Let $n \geq 3$. We classify the finite groups which are realised as subgroups of the sphere braid group $B_{n}\left(\mathbb{S}^{2}\right)$. Such groups must be of cohomological period 2 or 4 . Depending on the value of $n$, we show that the following are the maximal finite subgroups of $B_{n}\left(\mathbb{S}^{2}\right): \mathbb{Z}_{2(n-1)}$; the dicyclic groups of order $4 n$ and $4(n-2)$; the binary tetrahedral group $\mathrm{T}^{*}$; the binary octahedral group $\mathrm{O}^{*}$; and the binary icosahedral group $\mathrm{I}^{*}$. We give geometric as well as some explicit algebraic constructions of these groups in $B_{n}\left(\mathbb{S}^{2}\right)$ and determine the number of conjugacy classes of such finite subgroups. We also reprove Murasugi's classification of the torsion elements of $B_{n}\left(\mathbb{S}^{2}\right)$ and explain how the finite subgroups of $B_{n}\left(\mathbb{S}^{2}\right)$ are related to this classification, as well as to the lower central and derived series of $B_{n}\left(\mathbb{S}^{2}\right)$.
\end{abstract}

20F36; 20F50, 20E45, 57M99

\section{Introduction}

The braid groups $B_{n}$ of the plane were introduced by E Artin in 1925 [2; 3]. Braid groups of surfaces were studied by Zariski [41]. They were later generalised by Fox to braid groups of arbitrary topological spaces via the following definition [16]. Let $M$ be a compact, connected surface, and let $n \in \mathbb{N}$. We denote the set of all ordered $n$-tuples of distinct points of $M$, known as the $n$-th configuration space of $M$, by:

$$
F_{n}(M)=\left\{\left(p_{1}, \ldots, p_{n}\right) \mid p_{i} \in M \text { and } p_{i} \neq p_{j} \text { if } i \neq j\right\} .
$$

Configuration spaces play an important rôle in several branches of mathematics and have been extensively studied; see Cohen and Gitler [9] and Fadell and Husseini [14], for example.

The symmetric group $S_{n}$ on $n$ letters acts freely on $F_{n}(M)$ by permuting coordinates. The corresponding quotient will be denoted by $D_{n}(M)$. The $n-t h$ pure braid group $P_{n}(M)$ (respectively the $n$-th braid group $B_{n}(M)$ ) is defined to be the fundamental group of $F_{n}(M)$ (respectively of $D_{n}(M)$ ). 
Together with the real projective plane $\mathbb{R} P^{2}$, the braid groups of the 2 -sphere $\mathbb{S}^{2}$ are of particular interest, notably because they have nontrivial centre (see Gillette and Van Buskirk [17] and the authors' work [24]), and torsion elements [34; 40]. Indeed, Van Buskirk showed that among the braid groups of compact, connected surfaces, $B_{n}\left(\mathbb{S}^{2}\right)$ and $B_{n}\left(\mathbb{R} P^{2}\right)$ are the only ones to have torsion [40]. Let us recall briefly some of the properties of $B_{n}\left(\mathbb{S}^{2}\right)$ —see the papers of Van Buskirk with Fadell [15], with Gillette [17] and alone [40] for more details.

If $\mathbb{D}^{2} \hookrightarrow \mathbb{S}^{2}$ is an embedding of a topological disc, there is a group homomorphism $\iota: B_{n} \longrightarrow B_{n}\left(\mathbb{S}^{2}\right)$ induced by the inclusion. If $\beta \in B_{n}$, we shall denote its image $\iota(\beta)$ simply by $\beta$. Then $B_{n}\left(\mathbb{S}^{2}\right)$ is generated by $\sigma_{1}, \ldots, \sigma_{n-1}$ which are subject to the following relations:

$$
\begin{array}{cc}
\sigma_{i} \sigma_{j}=\sigma_{j} \sigma_{i} & \text { if }|i-j| \geq 2 \text { and } 1 \leq i, j \leq n-1, \\
\sigma_{i} \sigma_{i+1} \sigma_{i}=\sigma_{i+1} \sigma_{i} \sigma_{i+1} & \text { if } 1 \leq i \leq n-2, \\
\sigma_{1} \cdots \sigma_{n-2} \sigma_{n-1}^{2} \sigma_{n-2} \cdots \sigma_{1}=1 .
\end{array}
$$

Consequently, $B_{n}\left(\mathbb{S}^{2}\right)$ is a quotient of $B_{n}$. The first three sphere braid groups are finite: $B_{1}\left(\mathbb{S}^{2}\right)$ is trivial, $B_{2}\left(\mathbb{S}^{2}\right)$ is cyclic of order 2 . The group $B_{3}\left(\mathbb{S}^{2}\right)$ is a $\mathrm{ZS}$-metacyclic group (a group whose Sylow subgroups, commutator subgroup and commutator quotient group are all cyclic) of order 12 , isomorphic to the semi-direct product $\mathbb{Z}_{3} \rtimes \mathbb{Z}_{4}$ of cyclic groups with nontrivial action, which in turn is isomorphic to the dicyclic group Dic $_{12}$ of order 12 . The abelianisation of $B_{n}\left(\mathbb{S}^{2}\right)$ is isomorphic to the cyclic group $\mathbb{Z}_{2(n-1)}$. The kernel of the associated projection $\xi: B_{n}\left(\mathbb{S}^{2}\right) \longrightarrow \mathbb{Z}_{2(n-1)}$ (which is defined by $\xi\left(\sigma_{i}\right)=\overline{1}$ for all $\left.1 \leq i \leq n-1\right)$ is the commutator subgroup $\Gamma_{2}\left(B_{n}\left(\mathbb{S}^{2}\right)\right)$. If $w \in B_{n}\left(\mathbb{S}^{2}\right)$ then $\xi(w)$ is the exponent sum (relative to the $\left.\sigma_{i}\right)$ of $w$ modulo $2(n-1)$.

Gillette and Van Buskirk showed that if $n \geq 3$ and $k \in \mathbb{N}$ then $B_{n}\left(\mathbb{S}^{2}\right)$ has an element of order $k$ if and only if $k$ divides one of $2 n, 2(n-1)$ or 2(n-2) [17]. The torsion elements of $B_{n}\left(\mathbb{S}^{2}\right)$ and $B_{n}\left(\mathbb{R} P^{2}\right)$ were later characterised by Murasugi [34]. For $B_{n}\left(\mathbb{S}^{2}\right)$, these elements are as follows:

Theorem 1.1 [34] Let $n \geq 3$. Then the torsion elements of $B_{n}\left(\mathbb{S}^{2}\right)$ are precisely powers of conjugates of the following three elements:

(1) $\alpha_{0}=\sigma_{1} \cdots \sigma_{n-2} \sigma_{n-1}$ (which is of order $2 n$ )

(2) $\alpha_{1}=\sigma_{1} \cdots \sigma_{n-2} \sigma_{n-1}^{2}$ (of order $2(n-1)$ )

(3) $\alpha_{2}=\sigma_{1} \cdots \sigma_{n-3} \sigma_{n-2}^{2}($ of order $2(n-2))$

The three elements $\alpha_{0}, \alpha_{1}$ and $\alpha_{2}$ are respectively $n-$ th, $(n-1)$-th and $(n-2)-$ th roots of $\Delta_{n}$, where $\Delta_{n}$ is the so-called "full twist" braid of $B_{n}\left(\mathbb{S}^{2}\right)$, defined by 
$\Delta_{n}=\left(\sigma_{1} \cdots \sigma_{n-1}\right)^{n}$. So $B_{n}\left(\mathbb{S}^{2}\right)$ admits finite cyclic subgroups isomorphic to $\mathbb{Z}_{2 n}$, $\mathbb{Z}_{2(n-1)}$ and $\mathbb{Z}_{2(n-2)}$. In [25], we showed that $B_{n}\left(\mathbb{S}^{2}\right)$ is generated by $\alpha_{0}$ and $\alpha_{1}$. If $n \geq 3, \Delta_{n}$ is the unique element of $B_{n}\left(\mathbb{S}^{2}\right)$ of order 2 , and it generates the centre of $B_{n}\left(\mathbb{S}^{2}\right)$. It is also the square of the Garside element (or "half twist") defined by:

$$
T_{n}=\left(\sigma_{1} \cdots \sigma_{n-1}\right)\left(\sigma_{1} \cdots \sigma_{n-2}\right) \cdots\left(\sigma_{1} \sigma_{2}\right) \sigma_{1} .
$$

For $n \geq 4, B_{n}\left(\mathbb{S}^{2}\right)$ is infinite. It is an interesting question as to which finite groups are realised as subgroups of $B_{n}\left(\mathbb{S}^{2}\right)$ (apart of course from the cyclic groups $\left\langle\alpha_{i}\right\rangle$ and their subgroups given in Theorem 1.1). Another question is the following: how many conjugacy classes are there in $B_{n}\left(\mathbb{S}^{2}\right)$ of a given abstract finite group? As a partial answer to the first question, we proved in [25] that $B_{n}\left(\mathbb{S}^{2}\right)$ contains an isomorphic copy of the finite group $B_{3}\left(\mathbb{S}^{2}\right)$ of order 12 if and only if $n \not \equiv 1(\bmod 3)$.

While studying the lower central and derived series of the sphere braid groups, we showed that $\Gamma_{2}\left(B_{4}\left(\mathbb{S}^{2}\right)\right)$ is isomorphic to a semi-direct product of $\mathcal{Q}_{8}$ by a free group of rank 2 [23]. After having proved this result, we noticed that the question of the realisation of $\mathcal{Q}_{8}$ as a subgroup of $B_{n}\left(\mathbb{S}^{2}\right)$ had been explicitly posed by R Brown [7] in connection with the Dirac string trick and the fact that the fundamental group of $\mathrm{SO}(3)$ is isomorphic to $\mathbb{Z}_{2}[13 ; 28 ; 35]$. The case $n=4$ was studied by J G Thompson [39]. In a previous paper, we provided a complete answer to this question:

Theorem 1.2 [26] Let $n \in \mathbb{N}, n \geq 3$.

(1) $B_{n}\left(\mathbb{S}^{2}\right)$ contains a subgroup isomorphic to $\mathcal{Q}_{8}$ if and only if $n$ is even.

(2) If $n$ is divisible by 4 then $\Gamma_{2}\left(B_{n}\left(\mathbb{S}^{2}\right)\right)$ contains a subgroup isomorphic to $\mathcal{Q}_{8}$.

As we also pointed out in [26], for all $n \geq 3$, the construction of $\mathcal{Q}_{8}$ may be generalised in order to obtain a subgroup $\left\langle\alpha_{0}, T_{n}\right\rangle$ of $B_{n}\left(\mathbb{S}^{2}\right)$ isomorphic to the dicyclic group $\operatorname{Dic}_{4 n}$ of order $4 n$.

It is thus natural to ask which other finite groups are realised as subgroups of $B_{n}\left(\mathbb{S}^{2}\right)$. One common property of the above subgroups is that they are finite periodic groups of cohomological period 2 or 4 . In fact, this is true for all finite subgroups of $B_{n}\left(\mathbb{S}^{2}\right)$. Indeed, by [25], the universal covering $X$ of $F_{n}\left(\mathbb{S}^{2}\right)$ is a finite-dimensional complex which has the homotopy type of $\mathbb{S}^{3}$ (we were recently informed by V Lin that $X$ is biholomorphic to the direct product of $\operatorname{SL}(2, \mathbb{C})$ by the Teichmüller space of the $n$-punctured Riemann sphere [31]). Thus any finite subgroup of $B_{n}\left(\mathbb{S}^{2}\right)$ acts freely on $X$, and so has period 2 or 4 by [6, Proposition 10.2, Section 10, Chapter VII]. Since $\Delta_{n}$ is the unique element of order 2 of $B_{n}\left(\mathbb{S}^{2}\right)$, and it generates the centre $Z\left(B_{n}\left(\mathbb{S}^{2}\right)\right)$, the Milnor property must be satisfied for any finite subgroup of $B_{n}\left(\mathbb{S}^{2}\right)$. Recall also that a 
finite periodic group $G$ satisfies the $p^{2}$-condition (if $p$ is prime and divides the order of $G$ then $G$ has no subgroup isomorphic to $\mathbb{Z}_{p} \times \mathbb{Z}_{p}$ ), which implies that a Sylow $p$-subgroup of $G$ is cyclic or generalised quaternion, as well as the $2 p$-condition (each subgroup of order $2 p$ is cyclic). The classification of finite periodic groups is given by the Suzuki-Zassenhaus theorem (see Adem and Milgram [1] and Thomas [38] for example), and thus provides a possible line of attack for the subgroup realisation problem. The periods of the different families of these groups were determined in a series of papers by Golasiński and Gonçalves $[18 ; 19 ; 20 ; 21 ; 22]$, and so in theory we may obtain a list of those of period 4 . A list of all periodic groups of period 4 is provided in [38]. However, in the current context, a more direct approach is obtained via the relationship between the braid groups and the mapping class groups of $\mathbb{S}^{2}$, which we shall now recall.

For $n \in \mathbb{N}$, let $\mathcal{M}_{0, n}$ denote the mapping class group of the $n$-punctured sphere. We allow the $n$ marked points to be permuted. If $n \geq 2$, a presentation of $\mathcal{M}_{0, n}$ is obtained from that of $B_{n}\left(\mathbb{S}^{2}\right)$ by adding the relation $\Delta_{n}=1[32 ; 33]$. In other words, we have the following central extension:

$$
1 \longrightarrow\left\langle\Delta_{n}\right\rangle \longrightarrow B_{n}\left(\mathbb{S}^{2}\right) \stackrel{p}{\longrightarrow} \mathcal{M}_{0, n} \longrightarrow 1 .
$$

If $n=2, B_{2}\left(\mathbb{S}^{2}\right) \cong \mathcal{M}_{0,2} \cong \mathbb{Z}_{2}$. For $n=3$, since $\mathcal{M}_{0,3} \cong S_{3}$, this short exact sequence does not split, and in fact for $n \geq 4$ it does not split either [17].

Following Birman, this exact sequence may also be obtained in the following manner [5]. Let $\mathcal{H}^{+}\left(\mathbb{S}^{2}\right)$ denote the group of orientation-preserving homeomorphisms of $\mathbb{S}^{2}$, and let $X \in D_{n}\left(\mathbb{S}^{2}\right)$. Then $\mathcal{H}^{+}\left(\mathbb{S}^{2}, X\right)=\left\{f \in \mathcal{H}^{+}\left(\mathbb{S}^{2}\right) \mid f(X)=X\right\}$ is a subgroup of $\mathcal{H}^{+}\left(\mathbb{S}^{2}\right)$, and we have a fibration $\mathcal{H}^{+}\left(\mathbb{S}^{2}, X\right) \longrightarrow \mathcal{H}^{+}\left(\mathbb{S}^{2}\right) \longrightarrow D_{n}\left(\mathbb{S}^{2}\right)$, where the basepoint of $D_{n}\left(\mathbb{S}^{2}\right)$ is taken to be $X$, and where the second map evaluates an element of $\mathcal{H}^{+}\left(\mathbb{S}^{2}\right)$ on $X$. The resulting long exact sequence in homotopy yields:

$$
\begin{aligned}
(1-2) \quad \cdots \longrightarrow \pi_{1}\left(\mathcal{H}^{+}\left(\mathbb{S}^{2}, X\right)\right) \longrightarrow \underbrace{\pi_{1}\left(\mathcal{H}^{+}\left(\mathbb{S}^{2}\right)\right)}_{\mathbb{Z}_{2}} \longrightarrow \underbrace{\pi_{1}\left(D_{n}\left(\mathbb{S}^{2}\right)\right)}_{B_{n}\left(\mathbb{S}^{2}\right)} \\
\stackrel{\partial}{\longrightarrow} \underbrace{\pi_{0}\left(\mathcal{H}^{+}\left(\mathbb{S}^{2}, X\right)\right)}_{\mathcal{M}_{0, n}} \longrightarrow \underbrace{\pi_{0}\left(\mathcal{H}^{+}\left(\mathbb{S}^{2}\right)\right)}_{=\{1\}}
\end{aligned}
$$

The homomorphism $\partial: B_{n}\left(\mathbb{S}^{2}\right) \longrightarrow \mathcal{M}_{0, n}$ is the boundary operator which we shall use in Section 3 in order to describe the geometric realisation of the finite subgroups of $B_{n}\left(\mathbb{S}^{2}\right)$. If $n \geq 3$ then $\pi_{1}\left(\mathcal{H}^{+}\left(\mathbb{S}^{2}, X\right)\right)=\{1\}[12 ; 27 ; 36]$, and we thus recover extension (1-1) (the interpretation of the Dirac string trick in terms of the sphere braid groups $[13 ; 28 ; 35]$ gives rise to the identification of $\pi_{1}\left(\mathcal{H}^{+}\left(\mathbb{S}^{2}\right)\right)$ with $\left.\left\langle\Delta_{n}\right\rangle\right)$. 
In a recent paper, Stukow applies Kerckhoff's solution of the Nielsen realisation problem [30] to classify the finite maximal subgroups of $\mathcal{M}_{0, n}$ [37]. Applying his results to extension (1-1), we shall see in Section 2 that their counterparts in $B_{n}\left(\mathbb{S}^{2}\right)$ are cyclic, dicyclic and binary polyhedral groups:

Theorem 1.3 Let $n \geq 3$. The maximal finite subgroups of $B_{n}\left(\mathbb{S}^{2}\right)$ are:

(1) $\mathbb{Z}_{2(n-1)}$ if $n \geq 5$.

(2) the dicyclic group $\mathrm{Dic}_{4 n}$ of order $4 n$.

(3) the dicyclic group $\operatorname{Dic}_{4(n-2)}$ if $n=5$ or $n \geq 7$.

(4) the binary tetrahedral group, denoted by $\mathrm{T}^{*}$, if $n \equiv 4(\bmod 6)$

(5) the binary octahedral group, denoted by $\mathrm{O}^{*}$, if $n \equiv 0,2(\bmod 6)$.

(6) the binary icosahedral group, denoted by $\mathrm{I}^{*}$, if $n \equiv 0,2,12,20(\bmod 30)$.

Remarks 1.4 (1) If $n$ is odd then the only finite subgroups of $B_{n}\left(\mathbb{S}^{2}\right)$ are cyclic or dicyclic. In the latter case, the dicyclic group $\mathrm{Dic}_{4 n}($ resp. Dic $4(n-2))$ is ZSmetacyclic [11], and is isomorphic to $\mathbb{Z}_{n} \rtimes \mathbb{Z}_{4}$ (resp. $\mathbb{Z}_{n-2} \rtimes \mathbb{Z}_{4}$ ), where the action is multiplication by -1 .

If $n$ is even then one of the binary tetrahedral or octahedral groups is realised as a maximal finite subgroup of $B_{n}\left(\mathbb{S}^{2}\right)$. Further, since $\mathrm{T}^{*}$ is a subgroup of $\mathrm{O}^{*}, \mathrm{~T}^{*}$ is realised as a subgroup of $B_{n}\left(\mathbb{S}^{2}\right)$ for all $n$ even, $n \geq 4$.

(2) The groups of Theorem 1.3 and their subgroups are the finite groups of quaternions [10]. Indeed, for $p, q, r \in \mathbb{N}$, let us denote

$$
\langle p, q, r\rangle=\left\langle A, B, C \mid A^{p}=B^{q}=C^{r}=A B C\right\rangle .
$$

Then $\mathbb{Z}_{2(n-1)}=\langle n-1, n-1,1\rangle, \operatorname{Dic}_{4 n}=\langle n, 2,2\rangle, \operatorname{Dic}_{4(n-2)}=\langle n-2,2,2\rangle, \mathrm{T}^{*}=$ $\langle 3,3,2\rangle, \mathrm{O}^{*}=\langle 4,3,2\rangle$ and $\mathrm{I}^{*}=\langle 5,3,2\rangle$. It is shown in Coxeter [10] and Coxeter and Moser [11] that for $\mathrm{T}^{*}, \mathrm{O}^{*}$ and $\mathrm{I}^{*}$, this presentation is equivalent to:

$$
\langle p, 3,2\rangle=\left\langle A, B \mid A^{p}=B^{3}=(A B)^{2}\right\rangle,
$$

for $p \in\{3,4,5\}$, and that the element $A^{p}$ is central and is the unique element of order 2 of $\langle p, 3,2\rangle$.

(3) Some finite subgroups of the braid groups and mapping class groups of the sphere were studied by D Benson and FCohen in connection with the homology and cohomology of subgroups of certain mapping class groups $[4 ; 8]$, notably those of orientable surfaces of genus 2 . 
In Section 2, we also generalise another result of Stukow concerning the conjugacy classes of finite subgroups of $\mathcal{M}_{0, n}$ to $B_{n}\left(\mathbb{S}^{2}\right)$ :

\section{Proposition 1.5}

(1) Two maximal finite subgroups of $B_{n}\left(\mathbb{S}^{2}\right)$ are isomorphic if and only if they are conjugate.

(2) Each abstract finite subgroup $G$ of $B_{n}\left(\mathbb{S}^{2}\right)$ is realised as a single conjugacy class within $B_{n}\left(\mathbb{S}^{2}\right)$, with the exception, when $n$ is even, of the following cases, for which there are precisely two conjugacy classes:

(a) $\quad G=\mathbb{Z}_{4}$.

(b) $G=$ Dic $_{4 r}$, where $r$ divides $n / 2$ or $(n-2) / 2$.

In Section 3, we explain how to obtain geometrically the subgroups of Theorem 1.3, and we also give explicit group presentations of the cyclic and dicyclic subgroups, as well as in the special case of $\mathrm{T}^{*}$ for $n=4$ and $n=6$.

In order to understand better the finite subgroups of $B_{n}\left(\mathbb{S}^{2}\right)$, it is often useful to know their relationship with the three classes of elements described in Theorem 1.1. This shall be carried out in Proposition 4.1 (see Section 4).

The two conjugacy classes of part (2)(a) of Proposition 1.5 are realised by the subgroups $\left\langle\alpha_{0}^{n / 2}\right\rangle$ and $\left\langle\alpha_{2}^{(n-2) / 2}\right\rangle$ (they are non conjugate since they project to nonconjugate subgroups in $S_{n}$ ). In Section 5, we construct the two conjugacy classes of part (2)(b):

Theorem 1.6 Let $n \geq 4$ be even. Let $N \in\{n, n-2\}$, and let $x=\alpha_{0}$ (resp. $x=$ $\alpha_{0} \alpha_{2} \alpha_{0}^{-1}$ ) if $N=n$ (resp. $N=n-2$ ). Set $N=2^{l} k$, where $l \in \mathbb{N}$, and $k$ is odd. Then for $j=0,1, \ldots, l$, and $q$ a divisor of $k$, we have the following:

(1) $B_{n}\left(\mathbb{S}^{2}\right)$ contains $2^{j}$ copies of $\operatorname{Dic}_{2^{l+2-j} k / q}$ of the form $\left\langle x^{2^{j} q}, x^{i q} T_{n}\right\rangle$, where $i=0,1, \ldots, 2^{j}-1$.

(2) If $0 \leq i, i^{\prime} \leq 2^{j}-1,\left\langle x^{2^{j} q}, x^{i q} T_{n}\right\rangle$ and $\left\langle x^{2^{j} q}, x^{i^{\prime} q} T_{n}\right\rangle$ are conjugate if and only if $i-i^{\prime}$ is even.

Another question arising from Theorem 1.2 is the existence of copies of $\mathcal{Q}_{8}$ lying in $\Gamma_{2}\left(B_{n}\left(\mathbb{S}^{2}\right)\right)$. More generally, one may ask whether the dicyclic groups constructed above (and indeed the other finite subgroups of $\left.B_{n}\left(\mathbb{S}^{2}\right)\right)$ are contained in $\Gamma_{2}\left(B_{n}\left(\mathbb{S}^{2}\right)\right)$. In the dicyclic case, we have the following result, also proved in Section 5: 
Proposition 1.7 Let $n \geq 4$ be even, let $N \in\{n, n-2\}$, and let $r$ divide $N$. If $r$ does not divide $N / 2$ then the subgroups of $B_{n}\left(\mathbb{S}^{2}\right)$ abstractly isomorphic to Dic $4 r$ are not contained in $\Gamma_{2}\left(B_{n}\left(\mathbb{S}^{2}\right)\right)$. If $r$ divides $N / 2$ then up to conjugacy, $B_{n}\left(\mathbb{S}^{2}\right)$ has a two subgroups abstractly isomorphic to Dic $c_{4 r}$, one of which is contained in $\Gamma_{2}\left(B_{n}\left(\mathbb{S}^{2}\right)\right)$, and the other not. In particular, $B_{n}\left(\mathbb{S}^{2}\right)$ exhibits the two conjugacy classes of $\mathcal{Q}_{8}$, one of which lies in $\Gamma_{2}\left(B_{n}\left(\mathbb{S}^{2}\right)\right)$, the other not.

The corresponding result for the binary polyhedral groups may be found in Proposition 5.1. As a corollary of our results we obtain an alternative proof of Theorem 1.1 (see Section 6).

Acknowledgements This work took place during the visits of the second author to the Departmento de Matemática do IME - Universidade de São Paulo during the periods 10-27 August 2007 and 4-23 October 2007, and of the visit of the first author to the Laboratoire de Mathématiques Emile Picard, Université Paul Sabatier, Toulouse, during the period 27 August-17 September 2007. It was supported by the international Cooperation USP/Cofecub project number 105/06, by an Aide Ponctuelle de Coopération from the Université Paul Sabatier, by the Pró-Reitoria de Pesquisa Projeto 1 and by the Projeto Temático FAPESP no. 2004/10229-6 "Topologia Algébrica, Geometrica e Diferencial". The authors would like to thank Professor F R Cohen for pointing out some relevant references.

\section{The classification of the finite maximal subgroups of $B_{n}\left(\mathbb{S}^{2}\right)$}

In this section, we prove Theorem 1.3. We start by making some remarks concerning the central extension (1-1). We denote the order of a finite group $G$ by $|G|$.

Remarks 2.1 Let $G$ be a finite subgroup of $B_{n}\left(\mathbb{S}^{2}\right)$.

(1) If $H$ is a finite subgroup of $\mathcal{M}_{0, n}$ then $p^{-1}(H)$ is a finite subgroup of $B_{n}\left(\mathbb{S}^{2}\right)$ of order $2|H|$.

(2) If $|G|$ is odd then $\Delta_{n} \notin G$, and so $G \cong p(G)$. Conversely, if $G \cong p(G)$ then $\left.p\right|_{G}$ is injective, and thus $\Delta_{n} \notin G$, so $|G|$ is odd.

(3) If $|G|$ is even then $\Delta_{n} \in G$, and so we obtain the following short exact sequence:

$$
1 \longrightarrow\left\langle\Delta_{n}\right\rangle \longrightarrow G \stackrel{\left.p\right|_{G}}{\longrightarrow} p(G) \longrightarrow 1,
$$

where $p(G)$ is a finite subgroup of $\mathcal{M}_{0, n}$ of order $|G| / 2$. 
(4) If $G$ is a maximal finite subgroup of $B_{n}\left(\mathbb{S}^{2}\right)$ then $|G|$ is even, and $p(G)$ is a maximal finite subgroup of $\mathcal{M}_{0, n}$. Conversely, if $H$ is a maximal finite subgroup of $\mathcal{M}_{0, n}$ then $p^{-1}(H)$ is a maximal finite subgroup of $B_{n}\left(\mathbb{S}^{2}\right)$.

We recall Stukow's theorem:

Theorem 2.2 [37] Let $n \geq 3$. The maximal finite subgroups of $\mathcal{M}_{0, n}$ are:

(1) $\mathbb{Z}_{n-1}$ if $n \neq 4$.

(2) the dihedral group $D_{2 n}$ of order $2 n$.

(3) the dihedral group $D_{2(n-2)}$ if $n=5$ or $n \geq 7$.

(4) $A_{4}$ if $n \equiv 4,10(\bmod 12)$.

(5) $S_{4}$ if $n \equiv 0,2,6,8,12,14,18,20(\bmod 24)$.

(6) $A_{5}$ if $n \equiv 0,2,12,20,30,32,42,50(\bmod 60)$.

Remark 2.3 In the case $n=3, \mathcal{M}_{0,3}$ is isomorphic to $\mathrm{D}_{6}$, obtained as a maximal subgroup in part (2) of Theorem 2.2, and so its subgroup isomorphic to $\mathbb{Z}_{2}$ is not maximal. This explains the discrepancy between the value of $n$ in part (1) of Theorems 1.3 and 2.2.

Proof of Theorem 1.3 By Remarks 2.1, we just need to check that the given groups are those obtained as extensions of $\left\langle\Delta_{n}\right\rangle$ by the groups of Theorem 2.2. We start by making some preliminary remarks. Let $H$ be one of the finite maximal subgroups of $\mathcal{M}_{0, n}$, and let $G$ be a finite (maximal) subgroup of $B_{n}\left(\mathbb{S}^{2}\right)$ of order $2|H|$ which fits into the following short exact sequence:

$$
1 \longrightarrow\left\langle\Delta_{n}\right\rangle \longrightarrow G \stackrel{\left.p\right|_{G}}{\longrightarrow} H \longrightarrow 1,
$$

where $\Delta_{n} \in G$ belongs to the centre of $G$, and is the unique element of $G$ of order 2 . Then $G=p^{-1}(H)$, and so is unique.

Suppose that $y \in H$ is of order $k \geq 2$. Then $y$ has two preimages in $G$, of the form $x$ and $x \Delta_{n}$, say, and $x$ is of order $k$ or $2 k$. If $k$ is even then by Remarks 2.1(3), $x$ must be of order $2 k, x^{k}=\Delta_{n}$ and $\Delta_{n} \in\langle x\rangle$. If $k$ is odd then $x$ is of order $k$ (resp. $2 k$ ) if and only if $x \Delta_{n}$ is of order $2 k$ (resp. $k$ ).

A presentation of $G$ may be obtained by applying standard results concerning the presentation of an extension [29, Theorem 1, Chapter 13]. If $H$ is generated by $h_{1}, \ldots, h_{k}$ then $G$ is generated by $g_{1}, \ldots, g_{k}, \Delta_{n}$, where $p\left(g_{i}\right)=h_{i}$ for $i=1, \ldots, k$. One relation of $G$ is just $\Delta_{n}^{2}=1$, that of $\operatorname{Ker}(p)$. Since $\operatorname{Ker}(p) \subseteq Z(G)$, the remaining 
relations of $G$ are obtained by rewriting the relators of $H$ in terms of the coset representatives, and expressing the corresponding element in the form $\Delta_{n}^{\varepsilon}$, where $\varepsilon \in\{0,1\}$.

We consider the six cases of Theorem 2.2 as follows.

(1) $H \cong \mathbb{Z}_{n-1}$ : let $y$ be a generator of $H$, and let $x \in G$ be such that $p(x)=y$. Then $G=\left\langle\Delta_{n}, x\right\rangle$ and $|G|=2(n-1)$. If $n$ is odd then $\Delta_{n} \in\langle x\rangle, G=\langle x\rangle$, and $x$ is of order $2(n-1)$. If $n$ is even then $G=\left\langle x \Delta_{n}\right\rangle$ (resp. $\left.G=\langle x\rangle\right)$ if $x$ is of order $n-1$ (resp. $2(n-1))$, and $G \cong \mathbb{Z}_{2(n-1)}$ in both cases.

(2) $H \cong \mathrm{D}_{2 n}$ : let $y, z \in H$ be such that $o(y)=n, o(z)=2$ and $z y z^{-1}=y^{-1}$, and let $x, w \in G$ be such that $p(x)=y$ and $p(w)=z$. So $G=\left\langle\Delta_{n}, x, w\right\rangle$ and $|G|=4 n$. From above, it follows that $w^{2}=\Delta_{n}$, so $G=\langle x, w\rangle$. If $n$ is even then $x$ is of order $2 n$ and $x^{n}=\Delta_{n}$. The same result may be obtained if $n$ is odd, replacing $x$ by $x \Delta_{n}$ if necessary. Further, $w x w^{-1} x \in \operatorname{Ker}(p)$. If $w x w^{-1} x=\Delta_{n}$ then $(w x)^{2}=1$. So either $w=x^{-1}$ or $w x=\Delta_{n}$, and in both cases we conclude that $G=\langle x\rangle$ which contradicts $|G|=4 n$. Hence $w x w^{-1} x=1$, and since $|G|=4 n, G$ is isomorphic to Dic $4 n$.

(3) $H \cong \mathrm{D}_{2(n-2)}$ : the previous argument shows that $G \cong \operatorname{Dic}_{4(n-2)}$.

(4) Suppose that $H$ is isomorphic to one of the remaining groups $A_{4}, S_{4}$ or $A_{5}$ of Theorem 2.2. Let $p=3$ if $H \cong A_{4}, p=4$ if $H \cong S_{4}$, and $p=5$ if $H \cong A_{5}$. Then $H$ has a presentation given by $[10 ; 11]$ :

$$
H=\left\langle u, v \mid u^{2}=v^{3}=(u v)^{p}=1\right\rangle .
$$

Let $x, w \in G$ be such that $p(x)=u$ and $p(w)=v$. Then $G=\left\langle x, w, \Delta_{n}\right\rangle$. From above, we must have $x^{2}=\Delta_{n}$. Further, replacing $w$ by $w \Delta_{n}$, we may suppose that $w^{3}=\Delta_{n}$. If $p=4$ then $(x w)^{p}=\Delta_{n}$, while if $p \in\{3,5\}$, replacing $x$ by $x \Delta_{n}$ if necessary, we may suppose that $(x w)^{p}=\Delta_{n}$. It is shown in [10;11] that $x^{2}=w^{3}=(x w)^{p}=\Delta_{n}$ implies that $\Delta_{n}^{2}=1$, so $G$ admits a presentation given by:

$$
G=\left\langle x, w \mid x^{2}=w^{3}=(x w)^{p}\right\rangle .
$$

Thus $G \cong \mathrm{T}^{*}$ if $p=3, G \cong \mathrm{O}^{*}$ if $p=4$ and $G \cong \mathrm{I}^{*}$ if $p=5$. This completes the proof of the theorem.

Remarks 2.4 Let $G_{1}, G_{2}$ be finite subgroups of $B_{n}\left(\mathbb{S}^{2}\right)$.

(1) If they are of odd order then by Remarks 2.1, $G_{1}$ and $G_{2}$ are isomorphic if and only if $p\left(G_{1}\right)$ and $p\left(G_{2}\right)$ are isomorphic. So suppose that $G_{1}$ and $G_{2}$ are of even order. If $p\left(G_{1}\right)$ and $p\left(G_{2}\right)$ are isomorphic then it follows from the construction of Theorem 1.3 that $G_{1}$ and $G_{2}$ are isomorphic. Conversely, suppose that $G_{1}$ and $G_{2}$ 
are isomorphic via an isomorphism $\alpha: G_{1} \longrightarrow G_{2}$. Since $\Delta_{n}$ belongs to both, and is the unique element of order 2, we must have $\alpha\left(\Delta_{n}\right)=\Delta_{n}$, and thus $\alpha$ induces an isomorphism $\tilde{\alpha}: p\left(G_{1}\right) \longrightarrow p\left(G_{2}\right)$ satisfying $\tilde{\alpha} \circ p=p \circ \alpha$.

(2) If $G_{1}, G_{2}$ are conjugate then clearly so are $p\left(G_{1}\right)$ and $p\left(G_{2}\right)$. Conversely, suppose that $p\left(G_{1}\right), p\left(G_{2}\right)$ are conjugate subgroups of $\mathcal{M}_{0, n}$. Then there exists $g \in \mathcal{M}_{0, n}$ such that $p\left(G_{2}\right)=g p\left(G_{1}\right) g^{-1}$. If $G_{1}$ and $G_{2}$ are of even order, the fact that Equation (1-1) is a central extension implies that $G_{1}, G_{2}$ are conjugate. If $G_{1}$ and $G_{2}$ are of odd order, let $L_{i}=p^{-1}\left(p\left(G_{i}\right)\right)$ for $i=1,2$. Then $\left[L_{i}: G_{i}\right]=2$, and it follows from the even order case that $L_{1}$ and $L_{2}$ are conjugate in $B_{n}\left(\mathbb{S}^{2}\right)$. But $L_{i}=G_{i} \amalg \Delta_{n} G_{i}$, and its odd order elements are precisely those of $G_{i}$. So the conjugacy between $L_{1}$ and $L_{2}$ must send $G_{1}$ onto $G_{2}$.

We are now able to prove Proposition 1.5.

Proof of Proposition 1.5 Part (1) follows from Remarks 2.1 and 2.4. To prove part (2), let $G_{1}, G_{2}$ be abstractly isomorphic finite subgroups of $B_{n}\left(\mathbb{S}^{2}\right)$, and for $i=1,2$, let $H_{i}=p\left(G_{i}\right)$. Then $H_{1} \cong H_{2}$ : if the $G_{i}$ are of odd order then $H_{i} \cong G_{i}$, so $H_{1} \cong H_{2}$, while if the $G_{i}$ are of even order, any isomorphism between them must send $\Delta_{n} \in G_{1}$ onto $\Delta_{n} \in G_{2}$, and so projects to an isomorphism between the $H_{i}$. From Remarks 2.4(2), $G_{1}$ and $G_{2}$ are conjugate if and only if $H_{1}$ and $H_{2}$ are, and so the number of conjugacy classes of subgroups of $B_{n}\left(\mathbb{S}^{2}\right)$ isomorphic to $G_{1}$ is the same as the number of conjugacy classes of subgroups of $\mathcal{M}_{0, n}$ isomorphic to $H_{1}$. The result follows from the proof of Theorem 1.3 by remarking that a subgroup of $\mathcal{M}_{0, n}$ isomorphic to $\mathbb{Z}_{2}$ (resp. $\mathrm{D}_{2 r}$ ) lifts to a subgroup of $B_{n}\left(\mathbb{S}^{2}\right)$ which is isomorphic to $\mathbb{Z}_{4}($ resp. Dic $4 r)$.

\section{Realisation of the maximal finite subgroups of $B_{n}\left(\mathbb{S}^{2}\right)$}

In this section, we analyse the geometric and algebraic realisations of the subgroups given in Theorem 1.3.

\subsection{The algebraic realisation of some finite subgroups of $B_{n}\left(\mathbb{S}^{2}\right)$}

The maximal cyclic and dicyclic subgroups of $B_{n}\left(\mathbb{S}^{2}\right)$ may be realised as follows:

(1) $\mathbb{Z}_{2(n-1)} \cong\left\langle\alpha_{1}\right\rangle$.

(2) $\operatorname{Dic}_{4 n} \cong\left\langle\alpha_{0}, T_{n}\right\rangle$ (see the authors' work [26]).

(3) The algebraic realisation of $\mathrm{Dic}_{4(n-2)}$ is given by the following proposition: 
Proposition 3.1 For all $n \geq 3$, the subgroup $\left\langle\alpha_{0} \alpha_{2} \alpha_{0}^{-1}, T_{n}\right\rangle$ of $B_{n}\left(\mathbb{S}^{2}\right)$ is isomorphic to $\operatorname{Dic}_{4(n-2)}$.

Proof Let $x=\alpha_{0} \alpha_{2} \alpha_{0}^{-1}$. We know that $x$ is of order 2(n-2), and that $x^{n-1}=$ $\Delta_{n}=T_{n}^{2}$. Further, by standard properties of the corresponding elements in $B_{n}$ [5], $\alpha_{0} \sigma_{i} \alpha_{0}^{-1}=\sigma_{i+1}$ for all $i=1, \ldots, n-2$, and $T_{n} \sigma_{i} T_{n}^{-1}=\sigma_{n-i}$ for all $i=1, \ldots, n-1$. Hence $x=\sigma_{2} \cdots \sigma_{n-2} \sigma_{n-1}^{2}$, and

$$
T_{n} x T_{n}^{-1}=\sigma_{n-2} \cdots \sigma_{2} \sigma_{1}^{2}=\sigma_{n-1}^{-2} \sigma_{n-2}^{-1} \cdots \sigma_{2}^{-1}=x^{-1} .
$$

Thus $\left\langle x, T_{n}\right\rangle$ is isomorphic to a quotient of $\operatorname{Dic}_{4(n-2)}$. But $T_{n} \notin\langle x\rangle$, so $\left\langle x, T_{n}\right\rangle$ contains the $2(n-2)+1$ distinct elements of $\langle x\rangle \cup\left\{T_{n}\right\}$, and the result follows.

Remark 3.2 In the special case $n=4$, the binary tetrahedral group $\mathrm{T}^{*}$ may be realised as follows. Let $y=\sigma_{1} \sigma_{3}^{-1}$. From [26], we know that $\left\langle y, T_{4}\right\rangle \cong \mathcal{Q}_{8}$. In $B_{4}\left(\mathbb{S}^{2}\right)$, we also have $\left(\sigma_{2} \sigma_{1}\right)^{3}=\left(\sigma_{2} \sigma_{3}\right)^{3}=\Delta_{4}=T_{4}^{2}$. Then $\left\langle\alpha_{1}^{2}\right\rangle \cong \mathbb{Z}_{3}$ acts on $\left\langle y, T_{4}\right\rangle$ as follows:

$$
\begin{aligned}
\alpha_{1}^{2} \cdot T_{4} \cdot \alpha_{1}^{-2} & =\alpha_{1}^{2}\left(T_{4} \alpha_{1}^{-2} T_{4}^{-1}\right) T_{4}=\alpha_{1}^{2}\left(\sigma_{1}^{-2} \sigma_{2}^{-1} \sigma_{3}^{-1}\right)^{2} T_{4}\left(\text { by the action of } T_{4}\right) \\
& =\alpha_{1}^{2}\left(\sigma_{2} \sigma_{3}\right)^{2} T_{4}\left(\text { using the surface relation of } B_{n}\left(\mathbb{S}^{2}\right)\right) \\
& =\left(\sigma_{1} \sigma_{2} \sigma_{3}^{2}\right)^{2} \cdot \sigma_{3}^{-1} \sigma_{2}^{-1} \cdot\left(\sigma_{2} \sigma_{3}\right)^{3} T_{4} \\
& =\sigma_{1} \sigma_{2} \sigma_{3} \sigma_{1} \sigma_{2} \sigma_{1} \cdot \sigma_{1}^{-1} \sigma_{2}^{-1} \sigma_{1}^{-1} \cdot \sigma_{3} \sigma_{1} \sigma_{2} \sigma_{3} \sigma_{2}^{-1} T_{4}^{3}\left(\text { as } T_{4}^{2}=\left(\sigma_{2} \sigma_{3}\right)^{3}\right) \\
& =T_{4} \sigma_{1}^{-1} \sigma_{2}^{-1} \sigma_{3} \sigma_{2} \sigma_{3} \sigma_{2}^{-1} T_{4}^{3}\left(\text { as } \sigma_{1} \text { commutes with } \sigma_{3}\right) \\
& =T_{4} \sigma_{1}^{-1} \sigma_{3} T_{4}^{3}(\text { by the Artin braid relations }) \\
& \left.=T_{4} y^{-1} T_{4}^{-1}=y \text { (by the action of } T_{4} \text { on } y\right) .
\end{aligned}
$$

Further,

$$
\begin{aligned}
\alpha_{1}^{2} \cdot y \cdot \alpha_{1}^{-2} & =\left(\sigma_{1}^{-1} \sigma_{2}^{-1}\right)^{2} \cdot \sigma_{1} \sigma_{3}^{-1} \cdot\left(\sigma_{2} \sigma_{1}\right)^{2} \\
& =\left(\sigma_{1}^{-1} \sigma_{2}^{-1}\right)^{2} \cdot \sigma_{3}^{-1} \sigma_{2}^{-1} \cdot\left(\sigma_{2} \sigma_{1}\right)^{3}\left(\text { as } \sigma_{1} \text { commutes with } \sigma_{3}\right) \\
& =\sigma_{1}^{-1} \sigma_{2}^{-1} \sigma_{1}^{-1} \cdot \sigma_{2}^{-1} \sigma_{3}^{-1} \sigma_{2}^{-1} \cdot T_{4}^{2}\left(\text { as } T_{4}^{2}=\left(\sigma_{2} \sigma_{1}\right)^{3}\right) \\
& =\sigma_{1}^{-1} \sigma_{2}^{-1} \sigma_{1}^{-1} \sigma_{3}^{-1} \sigma_{2}^{-1} \sigma_{3}^{-1} \cdot T_{4}^{2} \text { (by the Artin braid relations) } \\
& =\sigma_{1}^{-1} \sigma_{2}^{-1} \sigma_{1}^{-1} \sigma_{3}^{-1} \sigma_{2}^{-1} \sigma_{1}^{-1} \cdot \sigma_{1} \sigma_{3}^{-1} T_{4}^{2} \\
& \left.=T_{4}^{-1} y T_{4}^{2}=T_{4} y \text { (since } T_{4}^{2} \text { is central }\right) .
\end{aligned}
$$

Hence $\mathrm{T}^{*}=\mathcal{Q}_{8} \rtimes \mathbb{Z}_{3} \cong\left\langle y, T_{4}\right\rangle \rtimes\left\langle\alpha_{1}^{2}\right\rangle$. 
Remark 3.3 We also have an algebraic representation of $\mathrm{T}^{*}$ in $B_{6}\left(\mathbb{S}^{2}\right)$. Let

$$
\begin{aligned}
& \gamma=\sigma_{5} \sigma_{4} \sigma_{1}^{-1} \sigma_{2}^{-1}, \\
& \delta=\sigma_{3}^{-1} \sigma_{4}^{-1} \sigma_{5}^{-1}\left(\sigma_{2}^{-1} \sigma_{1}^{-1} \sigma_{2}^{-1}\right) \sigma_{5} \sigma_{4} \sigma_{3} .
\end{aligned}
$$

Then we claim that $\langle\gamma, \delta\rangle \cong \mathcal{Q}_{8} \rtimes \mathbb{Z}_{3} \cong \mathrm{T}^{*}$, where the action permutes the elements $i, j, k$ of $\mathcal{Q}_{8}$. First, $\gamma^{3}=\delta^{2}=\Delta_{6}$. We now consider the subgroup $H=\left\langle\delta, \gamma \delta \gamma^{-1}\right\rangle$. The action of conjugation by $\gamma$ permutes cyclically the elements $\delta, \gamma \delta \gamma^{-1}$ and $\gamma \delta^{2} \gamma^{-1}$, so is compatible with the action of $\mathbb{Z}_{3}$ on $\mathcal{Q}_{8}$. It just remains to show that $H \cong \mathcal{Q}_{8}$. Clearly $\delta^{2}=\left(\gamma \delta \gamma^{-1}\right)^{2}=\Delta_{6}$. Let us now prove that

$$
\delta^{-1} \cdot \gamma \delta \gamma^{-1} \cdot \delta=\gamma \delta^{-1} \gamma^{-1}
$$

Set $\rho=\sigma_{5} \sigma_{4} \sigma_{3}, \gamma^{\prime}=\rho \gamma \rho^{-1}$ and $\delta^{\prime}=\rho \delta \rho^{-1}$. Then the above equation is equivalent in turn to the following relations:

$$
\begin{aligned}
& \delta^{\prime-1} \cdot \gamma^{\prime} \delta^{\prime} \gamma^{\prime-1} \cdot \delta^{\prime}=\gamma^{\prime} \delta^{\prime-1} \gamma^{\prime-1} \\
& \delta^{\prime-1} \gamma^{\prime} \delta^{\prime} \gamma^{\prime-1} \delta^{\prime 2} \delta^{\prime-1} \gamma^{\prime} \delta^{\prime} \gamma^{\prime-1}=1 \\
& {\left[\delta^{\prime-1}, \gamma^{\prime}\right]^{2}=\delta^{\prime-2}=\Delta_{6} .}
\end{aligned}
$$

We shall show that the latter relation holds. Notice that

$$
\gamma^{\prime}=\sigma_{5} \sigma_{4} \sigma_{3} \sigma_{5} \sigma_{4} \sigma_{1}^{-1} \sigma_{2}^{-1} \sigma_{3}^{-1} \sigma_{4}^{-1} \sigma_{5}^{-1}=\sigma_{5} \sigma_{4} \sigma_{5} \sigma_{3} \sigma_{4} \alpha_{0}
$$

Setting $\tau=\alpha_{0} \sigma_{5} \alpha_{0}^{-1}$, we have that:

$$
\begin{aligned}
{\left[\delta^{\prime-1}, \gamma^{\prime}\right]=} & \sigma_{5}^{-1} \sigma_{4}^{-1} \sigma_{5}^{-1} \sigma_{2} \sigma_{1} \sigma_{2} \cdot \sigma_{5} \sigma_{4} \sigma_{5} \sigma_{3} \sigma_{4} \alpha_{0} \cdot \sigma_{2}^{-1} \sigma_{1}^{-1} \sigma_{2}^{-1} \sigma_{5} \sigma_{4} \sigma_{5} . \\
& \alpha_{0}^{-1} \sigma_{4}^{-1} \sigma_{3}^{-1} \sigma_{5}^{-1} \sigma_{4}^{-1} \sigma_{5}^{-1} \\
= & \sigma_{2} \alpha_{0} \sigma_{5}^{-1} \alpha_{0} \sigma_{2}^{-1} \sigma_{1}^{-1} \sigma_{2}^{-1} \sigma_{5} \sigma_{4} \sigma_{5} \sigma_{5} \sigma_{4} \sigma_{3} \sigma_{2} \sigma_{1} \sigma_{4}^{-1} \sigma_{3}^{-1} \sigma_{5}^{-1} \sigma_{4}^{-1} \sigma_{5}^{-1} \\
= & \sigma_{2} \alpha_{0} \sigma_{5}^{-1} \alpha_{0} \sigma_{2}^{-1} \sigma_{1}^{-1} \sigma_{2}^{-1} \sigma_{5} \sigma_{3}^{-1} \sigma_{2}^{-1} \sigma_{1}^{-1} \sigma_{4}^{-1} \sigma_{3}^{-1} \sigma_{5}^{-1} \sigma_{4}^{-1} \sigma_{5}^{-1} \\
= & \sigma_{2} \alpha_{0} \sigma_{5}^{-1} \alpha_{0} \sigma_{2}^{-1} \sigma_{5} \sigma_{1}^{-1} \sigma_{2}^{-1} \sigma_{3}^{-1} \sigma_{4}^{-1} \sigma_{5}^{-1} \sigma_{1} \sigma_{1}^{-1} \sigma_{2}^{-1} \sigma_{1}^{-1} \sigma_{3}^{-1} \sigma_{4}^{-1} \sigma_{5}^{-1} \\
= & \sigma_{2} \alpha_{0} \sigma_{5}^{-1} \alpha_{0} \sigma_{2}^{-1} \sigma_{5} \alpha_{0} \sigma_{1} \sigma_{2}^{-1} \alpha_{0} \\
= & \sigma_{2} \alpha_{0} \sigma_{5}^{-1} \alpha_{0}^{-1} \alpha_{0}^{2} \sigma_{2}^{-1} \sigma_{5} \alpha_{0}^{-2} \alpha_{0}^{3} \sigma_{1} \sigma_{2}^{-1} \alpha_{0}^{-3} \alpha_{0}^{4} \\
= & \sigma_{2} \tau^{-1} \sigma_{4}^{-1} \sigma_{1} \sigma_{4} \sigma_{5}^{-1} \alpha_{0}^{4}=\sigma_{2} \tau^{-1} \sigma_{1} \sigma_{5}^{-1} \alpha_{0}^{4},
\end{aligned}
$$

since conjugation by $\alpha_{0}$ permutes cyclically the elements $\sigma_{1}, \sigma_{2}, \sigma_{3}, \sigma_{4}, \sigma_{5}$ and $\tau$. Thus

$$
\left[\delta^{\prime-1}, \gamma^{\prime}\right]^{2}=\sigma_{2} \tau^{-1} \sigma_{1} \sigma_{5}^{-1} \alpha_{0}^{4} \sigma_{2} \tau^{-1} \sigma_{1} \sigma_{5}^{-1} \alpha_{0}^{-4} \alpha_{0}^{8}=\sigma_{2} \tau^{-1} \sigma_{1} \sigma_{5}^{-1} \tau \sigma_{4}^{-1} \sigma_{5} \sigma_{3}^{-1} \alpha_{0}^{8} .
$$


Let $\xi=\sigma_{2} \tau^{-1} \sigma_{1} \sigma_{5}^{-1} \tau \sigma_{4}^{-1} \sigma_{5} \sigma_{3}^{-1}$. To prove that $\left[\delta^{\prime-1}, \gamma^{\prime}\right]^{2}=\Delta_{6}=\alpha_{0}^{6}$, it suffices to show that $\xi \alpha_{0}^{2}=1$. Now

$$
\begin{aligned}
\xi \alpha_{0}^{2} & =\sigma_{2} \tau^{-1} \sigma_{1} \sigma_{5}^{-1} \tau \sigma_{4}^{-1} \sigma_{5} \sigma_{3}^{-1} \alpha_{0}^{2}=\sigma_{2} \alpha_{0} \sigma_{5}^{-1} \alpha_{0}^{-1} \sigma_{1} \sigma_{5}^{-1} \alpha_{0} \sigma_{5} \alpha_{0}^{-1} \sigma_{4}^{-1} \sigma_{5} \sigma_{3}^{-1} \alpha_{0}^{2} \\
& =\sigma_{2} \alpha_{0} \sigma_{5}^{-1} \alpha_{0} \sigma_{5} \alpha_{0}^{-1} \sigma_{4}^{-1} \sigma_{5} \sigma_{3}^{-1} \sigma_{4} \sigma_{2}^{-1} \alpha_{0}=\sigma_{2} \alpha_{0} \sigma_{5}^{-1} \alpha_{0} \sigma_{5} \sigma_{3}^{-1} \sigma_{4} \sigma_{2}^{-1} \sigma_{3} \sigma_{1}^{-1} \\
& =\sigma_{2} \sigma_{1} \sigma_{2} \sigma_{3} \sigma_{4} \sigma_{1} \sigma_{2} \sigma_{3} \sigma_{4} \sigma_{5}^{2} \sigma_{4} \sigma_{3} \sigma_{3}^{-1} \sigma_{4}^{-1} \sigma_{3}^{-1} \sigma_{4} \sigma_{2}^{-1} \sigma_{3} \sigma_{1}^{-1} \\
& =\sigma_{1} \sigma_{2} \sigma_{1} \sigma_{3} \sigma_{4} \sigma_{1}^{-1} \sigma_{2}^{-1} \sigma_{4}^{-1} \sigma_{3}^{-1} \sigma_{2}^{-1} \sigma_{3} \sigma_{1}^{-1}=1 .
\end{aligned}
$$

This proves the claim, so $\langle\gamma, \delta\rangle \cong \mathrm{T}^{*}$.

\subsection{The geometric realisation of the finite subgroups of $B_{n}\left(\mathbb{S}^{2}\right)$}

The geometric realisation of the finite subgroups may be obtained by letting the corresponding subgroup of $\mathcal{M}_{0, n}$ act on the sphere with the $n$ strings attached in an appropriate manner. For the subgroups $\operatorname{Dic}_{4 n}, \mathbb{Z}_{2(n-1)}$ and $\operatorname{Dic}_{4(n-2)}$, we attach strings to $n$ symmetrically distributed points (resp. $n-1, n-2$ points) on the equator, and 0 (resp. 1,2) points at the poles. For $\mathrm{T}^{*}, \mathrm{O}^{*}$ and $\mathrm{I}^{*}$, the $n$ strings are attached symmetrically with respect to the associated regular polyhedron (for the values of $n$ given by Theorem 1.3) in the following manner.

(4) Let $H=A_{4}$ be the group of orientation-preserving symmetries of the tetrahedron. Then $n=6 k+4, k \geq 0$, and we take $k$ equally spaced points in the interior of each edge, plus one point at each vertex (or face).

(5) Let $H=S_{4}$ be the group of orientation-preserving symmetries of the cube (or octahedron).

(a) $n=12 k, k \in \mathbb{N}$ : take $k$ equally spaced points in the interior of each edge.

(b) $n=12 k+2, k \in \mathbb{N}$ : take $k-1$ equally spaced points in the interior of each edge, plus one point at each vertex and on each face.

(c) $n=12 k+6, k \geq 0$ : take $k$ equally spaced points in the interior of each edge, plus one point on each face.

(d) $n=12 k+8, k \geq 0$ : take $k$ equally spaced points in the interior of each edge, plus one point at each vertex.

(6) Let $H=A_{5}$ be the group of orientation-preserving symmetries of the dodecahedron (or icosahedron), which has 12 faces, 30 edges and 20 vertices. 
(a) $n=30 k, k \in \mathbb{N}$ : take $k$ equally spaced points in the interior of each edge.

(b) $n=30 k+2, k \in \mathbb{N}$ : take $k-1$ equally spaced points in the interior of each edge, plus one point at each vertex and on each face.

(c) $n=30 k+12, k \geq 0$ : take $k$ equally spaced points in the interior of each edge, plus one point on each face.

(d) $n=30 k+20, k \geq 0$ : take $k$ equally spaced points in the interior of each edge, plus one point at each vertex.

In each case, the action of the given group $H$ of symmetries yields the corresponding maximal finite subgroup of $B_{n}\left(\mathbb{S}^{2}\right)$. This follows essentially from the definition of the boundary operator $\partial: \pi_{1}\left(D_{n}\left(\mathbb{S}^{2}\right)\right) \longrightarrow \pi_{0}\left(\mathcal{H}^{+}\left(\mathbb{S}^{2}, X\right)\right)$ in the long exact sequence (1-2) which we now describe in detail in our setting. As in Section 1, let $X$ be the basepoint in $D_{n}\left(\mathbb{S}^{2}\right)$, and let $\psi: \mathcal{H}^{+}\left(\mathbb{S}^{2}\right) \longrightarrow D_{n}\left(\mathbb{S}^{2}\right)$ denote evaluation on $X$. So if $g \in \mathcal{H}^{+}\left(\mathbb{S}^{2}\right)$ then $\psi(g)=g(X)$. Let $\operatorname{Id}_{\mathbb{S}^{2}}$ be the basepoint in $\mathcal{H}^{+}\left(\mathbb{S}^{2}\right)$, so that $\psi\left(\operatorname{Id}_{\mathbb{S}^{2}}\right)=X$. Let $\beta \in B_{n}\left(\mathbb{S}^{2}\right)$ be a braid, and let $f:[0,1] \longrightarrow D_{n}\left(\mathbb{S}^{2}\right)$ be a geometric braid which represents $\beta$. So $f(0)=f(1)=X$, and the loop class $\langle f\rangle$ in $B_{n}\left(\mathbb{S}^{2}\right)$ is equal to $\beta$. Then $f$ lifts to $\tilde{f}:[0,1] \longrightarrow \mathcal{H}^{+}\left(\mathbb{S}^{2}\right)$ which satisfies $\tilde{f}(0)=\operatorname{Id}_{\mathbb{S}^{2}}$ and $\psi \circ \tilde{f}=f$. Hence $\psi \circ \tilde{f}(1)=f(1)=X$, and thus $\tilde{f}(1)$ belongs to the fibre $\mathcal{H}^{+}\left(\mathbb{S}^{2}, X\right)$. Geometrically, $\tilde{f}$ is an isotopy of $\mathbb{S}^{2}$ which realises $\beta$ on the points of $X$. Neither $\tilde{f}$ nor the corresponding endpoint $\tilde{f}(1)$ are unique, however all of the possible $\tilde{f}(1)$ belong to the same connected component of $\mathcal{H}^{+}\left(\mathbb{S}^{2}, X\right)$, and so determine a unique element, denoted $[\tilde{f}(1)]$, of $\pi_{0}\left(\mathcal{H}^{+}\left(\mathbb{S}^{2}, X\right)\right)$, which is the image under $\partial$ of $\beta$. Thus, if $\tilde{f}$ is an isotopy of $\mathbb{S}^{2}$ that realises $\beta, \partial(\beta)$ is the mapping class of the homeomorphism $\tilde{f}(1)$, and corresponds geometrically to just remembering the final homeomorphism (in particular, one forgets the strings of $\beta$ ).

Conversely, if $g \in \mathcal{H}^{+}\left(\mathbb{S}^{2}\right)$ satisfies $g(X)=X$, let $h:[0,1] \longrightarrow \mathcal{H}^{+}\left(\mathbb{S}^{2}\right)$ be an isotopy from $h(0)=\operatorname{Id}_{\mathbb{S}^{2}}$ to $h(1)=g$. Then $\psi \circ h$ is a loop in $D_{n}\left(\mathbb{S}^{2}\right)$ based at $X$, so describes a geometric braid obtained by attaching strings at the points of $X$ and following the isotopy $h$. In $\mathbb{S}^{2} \times[0,1]$, the strings are given by $\{(\psi \circ h(t), t)\}_{t \in[0,1]}=$ $\{(h(t)(X), t)\}_{t \in[0,1]}$. Thus $\langle\psi \circ h\rangle \in B_{n}\left(\mathbb{S}^{2}\right)$ is a braid, and by the above construction, $\partial(\langle\psi \circ h\rangle)=[h(1)]=[g]$. In other words, a choice of isotopy $h$ between the identity and $g \in \mathcal{H}^{+}\left(\mathbb{S}^{2}, X\right)$ allows us to lift the mapping class $[g]$ to a preimage $\beta=\langle\psi \circ h\rangle$ under $\partial$ which is obtained geometrically by attaching strings to $X$ during the isotopy $h$.

Let $r:[0,1] \longrightarrow \mathcal{H}^{+}\left(\mathbb{S}^{2}\right)$ denote rigid rotation through an angle $2 \pi$. So $r(0)=r(1)=$ $\operatorname{Id}_{\mathbb{S}^{2}}$, the loop class $\langle r\rangle$ generates $\pi_{1}\left(\mathcal{H}^{+}\left(\mathbb{S}^{2}\right)\right) \cong \mathbb{Z}_{2}$, and thus $\langle\psi \circ r\rangle=\psi_{*}(\langle r\rangle)=\Delta_{n}$ since $\psi_{*}: \pi_{1}\left(\mathcal{H}^{+}\left(\mathbb{S}^{2}\right)\right) \longrightarrow B_{n}\left(\mathbb{S}^{2}\right)$ is injective. The second preimage of $[g]$ under 
$\partial$ is obtained by considering the isotopy $h^{\prime}:[0,1] \longrightarrow \mathcal{H}^{+}\left(\mathbb{S}^{2}\right)$ that is the isotopy $h$ followed by $r$. The braids $\langle\psi \circ h\rangle$ and $\left\langle\psi \circ h^{\prime}\right\rangle$ differ by $\langle\psi \circ r\rangle=\Delta_{n}$, and thus define the two preimages of $[g]$ under $\partial$.

Finally, each finite subgroup $H$ of $\mathcal{M}_{0, n}$ is realised by a finite subgroup of isometries of $\mathbb{S}^{2}$ (which are the finite subgroups of $\mathrm{SO}(3)$ ) [30]. Each element of $H$ admits two preimages in $B_{n}\left(\mathbb{S}^{2}\right)$ which differ by $\Delta_{n}$. These preimages thus make up the finite subgroup $\partial^{-1}(H)$ of $B_{n}\left(\mathbb{S}^{2}\right)$ whose order is twice that of $H$.

\section{Position of the finite subgroups of $B_{n}\left(\mathbb{S}^{2}\right)$ relative to Mura- sugi's classification}

Let $n \geq 4$ be even. For $i=0,1,2$, let $G_{i}$ be the set of torsion elements of $B_{n}\left(\mathbb{S}^{2}\right)$ whose order divides $2(n-i)$. Equivalently, by Theorem 1.1, $G_{i}$ is the set of conjugates of powers of $\alpha_{i}$. Notice that $G_{i}$ is invariant under conjugation, $G_{i} \cap G_{j}=\left\langle\Delta_{n}\right\rangle$ for all $0 \leq i<j \leq 2$, and $G_{0} \cup G_{1} \cup G_{2}$ is the set of torsion elements of $B_{n}\left(\mathbb{S}^{2}\right)$. For many purposes, it is often useful to know where a finite subgroup $H$ of $B_{n}\left(\mathbb{S}^{2}\right)$ lies relative to the $G_{i}$. In this section, we carry out this calculation for all such subgroups.

Proposition 4.1 Let $H$ be a finite subgroup of $B_{n}\left(\mathbb{S}^{2}\right)$ of order greater than or equal to 3 .

(1) Suppose that $H$ is cyclic.

(a) If $|H|=4$ and $n$ is even then there exists a subgroup $H^{\prime}$ of $B_{n}\left(\mathbb{S}^{2}\right)$ isomorphic to $\mathbb{Z}_{4}$ nonconjugate to $H$. One of $H, H^{\prime}$ lies in $G_{0}$, while the other lies in $G_{2}$.

(b) If either $|H|=4$ and $n$ is odd, or if $|H| \neq 4$ then $H \subset G_{i}$, where $|H| \mid$ $2(n-i)$, and $i \in\{0,1,2\}$.

(2) Suppose that $H$ is a subgroup of a maximal noncyclic subgroup of $B_{n}\left(\mathbb{S}^{2}\right)$.

(a) If $H$ is a noncyclic subgroup contained in $\mathrm{Dic}_{4 n}$ or $\mathrm{Dic}_{4(n-2)}$ then it is itself dicyclic, of the form $\mathrm{Dic}_{4 k}$, where $k>1$ divides $n$ or $n-2$ respectively. Further:

(i) If $n$ is odd then $H \subset G_{i} \cup G_{1}$, where $i \in\{0,2\}$ and $|H| \mid 4(n-i)$.

(ii) Suppose that $n$ is even.

(A) If $k \mid n$ (resp. $k \mid n-2)$ but $k \nmid(n / 2)($ resp. $k \nmid((n-2) / 2))$ then $H$ lies in $G_{0} \cup G_{2}$ and meets both $G_{0}$ and $G_{2}$.

(B) If $k \mid(n / 2)($ resp. $k \mid((n-2) / 2))$ then there exists another subgroup $H^{\prime}$ of $B_{n}\left(\mathbb{S}^{2}\right)$ isomorphic to Dic $4 k$ but non conjugate to $H$. In this case, one of $H, H^{\prime}$ is contained wholly within $G_{0}$ (resp. $G_{2}$ ), and the other lies in $G_{0} \cup G_{2}$ and meets both $G_{0}$ and $G_{2}$. 
(b) Suppose that $H$ is a subgroup of a copy of $\mathrm{T}^{*}$ in the case that $\mathrm{T}^{*}$ is maximal.

(i) If $H \cong \mathrm{T}^{*}$ then $H$ lies in $G_{0} \cup G_{1}\left(\right.$ resp. $\left.G_{2} \cup G_{1}\right)$ if $n \equiv 4(\bmod 12)$ $(\operatorname{resp} . n \equiv 10(\bmod 12))$, and meets both $G_{0}\left(\operatorname{resp} . G_{2}\right)$ and $G_{1}$.

(ii) If $H$ is isomorphic to $\mathbb{Z}_{3}$ or $\mathbb{Z}_{6}$ then it is contained in $G_{1}$.

(iii) If $H$ is isomorphic to $\mathbb{Z}_{4}$ or $\mathcal{Q}_{8}$ then it is contained in $G_{0}$ if $n \equiv 4$ $(\bmod 12)$, and in $G_{2}$ if $n \equiv 10(\bmod 12)$.

(c) Suppose that $H$ is a subgroup of a copy of $\mathrm{I}^{*}$ in the case that $\mathrm{I}^{*}$ is maximal.

(i) If $H$ is isomorphic to I* then $H$ is contained in $G_{0}$ (resp. $\left.G_{2}\right)$ if $n \equiv 0$ $(\bmod 60)($ resp. $n \equiv 2(\bmod 60))$, and lies in $G_{0} \cup G_{2}$ and meets both $G_{0}$ and $G_{2}$ if $n \equiv 12,20,30,32,42,50(\bmod 60)$.

(ii) If $H$ is isomorphic to $\mathbb{Z}_{3}$ or $\mathbb{Z}_{6}$ then it is contained in $G_{0}$ if $n \equiv 0,12$ $(\bmod 30)$, and in $G_{2}$ if $n \equiv 2,20(\bmod 30)$.

(iii) If $H$ is isomorphic to $\mathbb{Z}_{5}$ or $\mathbb{Z}_{10}$ then it is contained in $G_{0}$ if $n \equiv 0,20$ $(\bmod 30)$, and in $G_{2}$ if $n \equiv 2,12(\bmod 30)$.

(iv) If $H$ is isomorphic to $\mathbb{Z}_{4}$ or $\mathcal{Q}_{8}$ then it is contained in $G_{0}$ if $n \equiv$ $0,12,20,32(\bmod 60)$, and in $G_{2}$ if $n \equiv 2,30,42,50(\bmod 60)$.

(v) If $H$ is isomorphic to $\mathrm{T}^{*}$ or to Dic 12 then it lies in $G_{0}$ if $n \equiv 0,12$ $(\bmod 60)$, in $G_{2}$ if $n \equiv 2,50(\bmod 60)$, and lies in $G_{0} \cup G_{2}$ and meets both $G_{0}$ and $G_{2}$ if $n \equiv 20,30,32,42(\bmod 60)$.

(vi) If $H$ is isomorphic to Dic 20 then it lies in $G_{0}$ if $n \equiv 0,20(\bmod 60)$, in $G_{2}$ if $n \equiv 2,42(\bmod 60)$, and lies in $G_{0} \cup G_{2}$ and meets both $G_{0}$ and $G_{2}$ if $n \equiv 12,30,32,50(\bmod 60)$.

(d) Suppose that $H$ is a subgroup of a copy of $\mathrm{O}^{*}$ in the case that $\mathrm{O}^{*}$ is maximal.

(i) If $H$ is isomorphic to $\mathrm{O}^{*}$ then it lies in $G_{0}$ if $n \equiv 0(\bmod 24)$, in $G_{2}$ if $n \equiv 2(\bmod 24)$, and lies in $G_{0} \cup G_{2}$ and meets both $G_{0}$ and $G_{2}$ if $n \equiv 6,8,12,14,18,20(\bmod 24)$.

(ii) If $H$ is isomorphic to $\mathrm{T}^{*}$ then it lies in $G_{0}$ if $n \equiv 0(\bmod 12)$, in $G_{2}$ if $n \equiv 2(\bmod 12)$, and lies in $G_{0} \cup G_{2}$ and meets both $G_{0}$ and $G_{2}$ if $n \equiv 6,8(\bmod 12)$.

(iii) If $H$ is isomorphic to $\mathcal{Q}_{16}$ then it lies in $G_{0}$ if $n \equiv 0,8(\bmod 24)$, in $G_{2}$ if $n \equiv 2,18(\bmod 24)$, and lies in $G_{0} \cup G_{2}$ and meets both $G_{0}$ and $G_{2}$ if $n \equiv 6,12,14,20(\bmod 24)$.

(iv) If $H$ is isomorphic to Dic 12 then it lies in $G_{0}$ if $n \equiv 0,6(\bmod 24)$, in $G_{2}$ if $n \equiv 2,20(\bmod 24)$, and lies in $G_{0} \cup G_{2}$ and meets both $G_{0}$ and $G_{2}$ if $n \equiv 8,12,14,18(\bmod 24)$.

(v) If $H$ is isomorphic to $\mathbb{Z}_{8}$ then it lies in $G_{0}$ if $n \equiv 0,8(\bmod 12)$, and in $G_{2}$ if $n \equiv 2,6(\bmod 12)$. 
(vi) If $H$ is isomorphic to $\mathbb{Z}_{4}$ then there exists another nonconjugate subgroup $H^{\prime}$ of $B_{n}\left(\mathbb{S}^{2}\right)$ isomorphic to $\mathbb{Z}_{4}$. One of $H, H^{\prime}$ is contained in $G_{0}$ if $n \equiv 0,8(\bmod 12)$, and in $G_{2}$ if $n \equiv 2,6(\bmod 12)$, while the other is contained in $G_{0}$ if $n \equiv 0,6,8,14(\bmod 24)$, and to $G_{2}$ if $n \equiv 2,12,18,20(\bmod 24)$.

(vii) If $H$ is isomorphic to $\mathcal{Q}_{8}$ then there exists another nonconjugate subgroup $H^{\prime}$ of $B_{n}\left(\mathbb{S}^{2}\right)$ isomorphic to $\mathcal{Q}_{8}$. One of $H, H^{\prime}$ is contained in $G_{0}$ if $n \equiv 0,8(\bmod 12)$, and to $G_{2}$ if $n \equiv 2,6(\bmod 12)$, while the other lies in $G_{0}$ if $n \equiv 0,8(\bmod 24)$, in $G_{2}$ if $n \equiv 2,18(\bmod 24)$, and lies in $G_{0} \cup G_{2}$ and meets both $G_{0}$ and $G_{2}$ if $n \equiv 6,12,14,20$ $(\bmod 24)$.

(viii) If $H$ is isomorphic to $\mathbb{Z}_{3}$ or $\mathbb{Z}_{6}$ then it lies in $G_{0}$ if $n \equiv 0(\bmod 6)$ and in $G_{2}$ if $n \equiv 2(\bmod 6)$.

Proof Let $H$ be a finite subgroup of $B_{n}\left(\mathbb{S}^{2}\right)$ of order at least three.

(1) Suppose first that $H$ is cyclic. Since $G_{i} \cap G_{j}=\left\langle\Delta_{n}\right\rangle$ and $\left|\left\langle\alpha_{i}\right\rangle\right|=2(n-i)$, the order of $H$ is sufficient to decide where $H$ lies, unless $n$ is even and $H$ is of order 4, in which case there is another nonconjugate subgroup $H^{\prime}$ isomorphic to $\mathbb{Z}_{4}$. One of $H, H^{\prime}$ is conjugate to $\left\langle\alpha_{0}^{n / 2}\right\rangle$ which is contained in $G_{0}$, while the other is conjugate to $\left\langle\alpha_{2}^{(n-2) / 2}\right\rangle$ which lies in $G_{2}$. These two cases may be distinguished easily by checking the permutation of a generator of $H, H^{\prime}$.

(2) Now suppose that $H$ is a subgroup of a maximal noncyclic subgroup of $B_{n}\left(\mathbb{S}^{2}\right)$. We consider the possible cases in turn.

(a) Firstly, let $H$ be a subgroup of the dicyclic group $\mathrm{Dic}_{4 n}$, which up to conjugation may be assumed to be $\left\langle\alpha_{0}, T_{n}\right\rangle=\left\langle\alpha_{0}\right\rangle \bigsqcup T_{n}\left\langle\alpha_{0}\right\rangle$. We first suppose that $n$ is odd. Then $\left\langle\alpha_{0}\right\rangle \subset G_{0}$, and the coset $T_{n}\left\langle\alpha_{0}\right\rangle$ consists of the elements of $\operatorname{Dic}_{4 n}$ of order 4 , so lies in $G_{1}$. The group $\operatorname{Dic}_{4 n}$ fits into the following short exact sequence:

$$
1 \longrightarrow \mathbb{Z}_{n} \longrightarrow \operatorname{Dic}_{4 n} \stackrel{g}{\longrightarrow} \mathbb{Z}_{4} \longrightarrow 1 \text {. }
$$

If $g(H)=\{\overline{0}\}$, then $H<\mathbb{Z}_{n}$, and $H$ is cyclic, of order dividing $n$, so lies in $G_{0}$. If $g(H)=\{\overline{0}, \overline{2}\}$, then $H<\mathbb{Z}_{2 n}$, and again $H$ is cyclic, of order dividing $2 n$, so lies in $G_{0}$. Finally, if $g(H)=\mathbb{Z}_{4}$ then we have

$$
1 \longrightarrow H \cap \mathbb{Z}_{n} \longrightarrow H \stackrel{g}{\longrightarrow} \mathbb{Z}_{4} \longrightarrow 1,
$$

and $H \cong \mathbb{Z}_{k} \rtimes \mathbb{Z}_{4}$, where $k$ divides $n$. If $k=1$ then $H \cong \mathbb{Z}_{4}$. Since $n$ is odd, $H$ must then lie in $G_{1}$. So suppose that $k>1$. Then $H=\left\langle\alpha_{0}^{n / k}, T_{n}\right\rangle$ is dicyclic, and so lies in $G_{0} \cup G_{1}$. 
Now suppose that $n$ is even. Then $\mathrm{Dic}_{4 n}$ fits into the following short exact sequence:

$$
1 \longrightarrow \mathbb{Z}_{2 n} \longrightarrow \operatorname{Dic}_{4 n} \stackrel{f}{\longrightarrow} \mathbb{Z}_{2} \longrightarrow 1 \text {. }
$$

If $f(H)=\{\overline{0}\}$ then $H \subset \mathbb{Z}_{2 n}$ and so lies in $G_{0}$. If $f(H)=\mathbb{Z}_{2}$ and $H \cap \mathbb{Z}_{2 n}$ were of odd order, then $H$ would be both dicyclic and of order twice an odd number, which cannot occur. So suppose that $f(H)=\mathbb{Z}_{2}$ and $H \cap \mathbb{Z}_{2 n}$ is of even order, $2 k$, say, where $k \mid n$. If $k=1$ then $H \cong \mathbb{Z}_{4}$, and $H$ may lie in $G_{0}$ or $G_{2}$ depending on the permutation of its generators. So suppose that $k \geq 2$. Then $H$ is dicyclic of order $4 k$. Now

$$
\operatorname{Dic}_{4 n}=\underbrace{\left\langle\alpha_{0}\right\rangle}_{\subset G_{0}} \coprod \underbrace{T_{n}\left\langle\alpha_{0}^{2}\right\rangle}_{\subset G_{0}} \coprod \underbrace{T_{n} \alpha_{0}\left\langle\alpha_{0}^{2}\right\rangle}_{\subset G_{2}} .
$$

The inclusions follow from the fact that the elements of $T_{n}\left\langle\alpha_{0}^{2}\right\rangle$ (resp. $T_{n} \alpha_{0}\left\langle\alpha_{0}^{2}\right\rangle$ ) are conjugate (in $\mathrm{Dic}_{4 n}$ ), $T_{n} \in G_{0}$, and

$$
\begin{aligned}
\pi\left(T_{n} \alpha_{0}\right) & =(1, n)(2, n-1) \cdots\left(\frac{n}{2}, \frac{n}{2}+1\right)(1, n, \ldots, 2) \\
& =(n)\left(\frac{n}{2}\right)(1, n-1)(2, n-2)(3, n-3) \cdots\left(\frac{n}{2}-1, \frac{n}{2}+1\right),
\end{aligned}
$$

where $\pi: B_{n}\left(\mathbb{S}^{2}\right) \longrightarrow S_{n}$ denotes the homomorphism defined on the generators by $\pi\left(\sigma_{i}\right)=(i, i+1)$. Thus $T_{n} \alpha_{0} \in G_{2}$.

If $k \nmid(n / 2)$ then by Proposition 1.5, there is just one conjugacy class of $\mathrm{Dic}_{4 k}$ of the form $\left\langle\alpha_{0}^{n / k}, T_{n}\right\rangle$, and since $n / k$ is odd, we have

$$
\operatorname{Dic}_{4 k}=\underbrace{\left\langle\alpha_{0}^{n / k}\right\rangle}_{\subset G_{0}} \coprod \underbrace{T_{n}\left\langle\alpha_{0}^{n / k}\right\rangle}_{\subset G_{2}} .
$$

In particular, all of the elements of $\operatorname{Dic}_{4 k}$ of order 4 belong to $G_{2}$. Thus we have $\operatorname{Dic}_{4 k} \cap\left(G_{0} \backslash G_{2}\right) \neq \varnothing$ and $\operatorname{Dic}_{4 k} \cap\left(G_{2} \backslash G_{0}\right) \neq \varnothing$.

If $k \mid(n / 2)$, by Proposition 1.5, there are two nonconjugate copies of $\mathrm{Dic}_{4 k}$ given by

$$
\left\langle\alpha_{0}^{n / k}, T_{n}\right\rangle=\underbrace{\left\langle\alpha_{0}^{n / k}\right\rangle}_{\subset G_{0}} \coprod \underbrace{T_{n}\left\langle\alpha_{0}^{n / k}\right\rangle}_{\subset G_{0}}
$$

and

$$
\left\langle\alpha_{0}^{n / k}, T_{n} \alpha_{0}\right\rangle=\underbrace{\left\langle\alpha_{0}^{n / k}\right\rangle}_{\subset G_{0}} \coprod \underbrace{T_{n} \alpha_{0}\left\langle\alpha_{0}^{n / k}\right\rangle}_{\subset G_{2}} .
$$

The first copy lies entirely within $G_{0}$, while the second lies in $G_{0} \cup G_{2}$ and meets both $G_{0} \backslash G_{2}$ and $G_{2} \backslash G_{0}$. 
A similar result holds for $\operatorname{Dic}_{4(n-2)}$ : its subgroups are either subgroups of $\mathbb{Z}_{2(n-2)}$, so lie in $G_{2}$, or else are dicyclic, of the form $\operatorname{Dic}_{4 k}$, where $k \mid n-2$. If $k=1$ then the subgroup in question is $\left\langle T_{n}\right\rangle$ which lies in $G_{0}$. If $k>1$ then as above, we distinguish two cases. If $k \nmid((n-2) / 2)$ then there is just one copy of $\operatorname{Dic}_{4 k}$ which lies in $G_{0} \cup G_{2}$ and meets both $G_{0} \backslash G_{2}$ and $G_{2} \backslash G_{0}$. If $k \mid((n-2) / 2)$, then setting $\alpha_{2}^{\prime}=\alpha_{0} \alpha_{2} \alpha_{0}^{-1}$, there are two copies of $\operatorname{Dic}_{4 k}$ : the first, $\left\langle\left(\alpha_{2}^{\prime}\right)^{n / k}\right\rangle, T_{n}$ lies in $G_{0} \cup G_{2}$ and meets both $G_{0} \backslash G_{2}$ and $G_{2} \backslash G_{0}$, and the second, $\left\langle\left(\alpha_{2}^{\prime}\right)^{n / k}, \alpha_{2}^{\prime} T_{n}\right\rangle$, is contained in $G_{2}$.

(b) Suppose that $H$ is a subgroup of a copy of $\mathrm{T}^{*}$ when $\mathrm{T}^{*}$ is maximal, so $n \equiv 4$ (mod 6). Assume first that $H \cong \mathrm{T}^{*}$. Since $H \cong \mathcal{Q}_{8} \rtimes \mathbb{Z}_{3}$, all of its order 4 elements are conjugate, and so all elements of $\mathcal{Q}_{8}$ must lie in the same $G_{i}$. Now $\mathcal{Q}_{8}=\mathrm{Dic}_{8}$, so from above, we must be in one of the cases $2 \mid(n / 2)$ or $2 \mid((n-2) / 2)$. Indeed if $n \equiv 4(\bmod 12)$ then $n=4+12 l=4(1+3 l), l \in \mathbb{N}$, and so $\mathcal{Q}_{8}$ is contained in $G_{0}$, while if $n \equiv 10(\bmod 12)$ then $n=10+12 l=2(5+6 l), l \in \mathbb{N}$, and so $\mathcal{Q}_{8}$ is contained in $G_{2}$. The remaining elements of $H$ are of order 3 or 6 , and since $n \equiv 4$ $(\bmod 6)$, lie in $G_{1}$. So if $n \equiv 4(\bmod 12)(\operatorname{resp} . n \equiv 10(\bmod 12))$ then $H$ lies in $G_{0} \cup G_{1}$ (resp. $G_{2} \cup G_{1}$ ) and meets both $G_{0}$ (resp. $G_{2}$ ) and $G_{1}$.

From this, we deduce immediately the following: if $H$ is isomorphic to $\mathbb{Z}_{3}$ or $\mathbb{Z}_{6}$ then it is contained in $G_{1}$, and if it is isomorphic to $\mathbb{Z}_{4}$ or $\mathcal{Q}_{8}$ then it is contained in $G_{0}$ if $n \equiv 4(\bmod 12)$, and in $G_{2}$ if $n \equiv 10(\bmod 12)$.

(c) Suppose that $H$ is a subgroup of a copy of $\mathrm{I}^{*}$ when $\mathrm{I}^{*}$ is maximal, so $n \equiv$ $0,2,12,20(\bmod 30)$. Assume first that $H \cong \mathrm{I}^{*}$. So $\mathrm{I}^{*}$ has a subgroup isomorphic to $\mathrm{T}^{*}$, whose copy of $\mathcal{Q}_{8}$ lies entirely in $G_{0}$ or $G_{2}$. The subgroups of order 8 of $H$ are its Sylow 2-subgroups, so are conjugate, and thus all lie either in $G_{0}$ or in $G_{2}$. Hence from the analysis of the dicyclic case, 2 divides $n / 2$ or $(n-2) / 2$. Further, all elements of $H$ of order 4 are contained in one of its subgroups isomorphic to $\mathcal{Q}_{8}$ (because the order 2 elements of $A_{5}$ are the product of two transpositions, and are contained in a subgroup isomorphic to $\mathbb{Z}_{2} \oplus \mathbb{Z}_{2}$, which lifts to $\mathcal{Q}_{8}$ in $\mathrm{I}^{*}$ ). Hence all order 4 elements of $H$ lie either in $G_{0}$ if $4 \mid n$, or in $G_{2}$ if $4 \mid n-2$. The remaining elements of $H$ are of order 3,6,5 and 10, and lie in either $G_{0}$ or $G_{2}$ depending on the value of $n$ modulo the order. Thus $H$ lies entirely in $G_{0}$ (resp. $G_{2}$ ) if $n \equiv 0$ $(\bmod 60)($ resp. $n \equiv 2(\bmod 60))$, and lies in $G_{0} \cup G_{2}$ and meets both $G_{0}$ and $G_{2}$ if $n \equiv 12,20,30,32,42,50(\bmod 60)$.

We now consider the other possibilities for subgroups of $\mathrm{I}^{*}$ : if $H$ is isomorphic to either $\mathbb{Z}_{3}$ or $\mathbb{Z}_{6}$, it is contained in $G_{0}$ if $n \equiv 0,12(\bmod 30)$, and in $G_{2}$ if $n \equiv 2,20$ $(\bmod 30)$; if $H$ is isomorphic to either $\mathbb{Z}_{5}$ or $\mathbb{Z}_{10}$, it is contained in $G_{0}$ if $n \equiv 0,20$ $(\bmod 30)$, and in $G_{2}$ if $n \equiv 2,12(\bmod 30)$; and if $H$ is isomorphic to either $\mathbb{Z}_{4}$ or $\mathcal{Q}_{8}$, it is contained in $G_{0}$ if $n \equiv 0,12,20,32(\bmod 60)$, and in $G_{2}$ if $n \equiv 2,30,42,50$ 
(mod 60). Next, if $H$ is isomorphic to $\mathrm{T}^{*}$, it consists of a copy of $\mathcal{Q}_{8}$ and elements of order 3 and 6 , so lies in $G_{0}$ if $n \equiv 0,12(\bmod 60)$, in $G_{2}$ if $n \equiv 2,50(\bmod 60)$, and lies in $G_{0} \cup G_{2}$ and meets both $G_{0}$ and $G_{2}$ if $n \equiv 20,30,32,42(\bmod 60)$. Now suppose that $H$ is isomorphic to $\operatorname{Dic}_{12} \cong \mathbb{Z}_{3} \rtimes \mathbb{Z}_{4}=\mathbb{Z}_{6} \amalg T_{n} \mathbb{Z}_{6}$. Since the elements of $T_{n} \mathbb{Z}_{6}$ are of order 4 , it follows from the analysis of the cyclic subgroups that $H$ satisfies the same conditions as in the case of $\mathrm{T}^{*}$. Finally, if $H$ is isomorphic to Dic $_{20} \cong \mathbb{Z}_{5} \rtimes \mathbb{Z}_{4}=\mathbb{Z}_{10} \bigsqcup T_{n} \mathbb{Z}_{10}$, since the elements of $T_{n} \mathbb{Z}_{10}$ are of order 4 , it follows from the analysis of the cyclic subgroups that $H$ lies in $G_{0}$ if $n \equiv 0,20$ $(\bmod 60)$, in $G_{2}$ if $n \equiv 2,42(\bmod 60)$, and lies in $G_{0} \cup G_{2}$ and meets both $G_{0}$ and $G_{2}$ if $n \equiv 12,30,32,50(\bmod 60)$.

(d) Suppose that $H$ is a subgroup of a copy of $\mathrm{O}^{*}$ when $\mathrm{O}^{*}$ is maximal, so $n \equiv 0,2$ (mod 6). Assume first that $H \cong \mathrm{O}^{*}$. Then it has a subgroup isomorphic to $\mathrm{T}^{*}$ (which is unique since $S_{4}$ has a unique subgroup abstractly isomorphic to $A_{4}$ ), and the copy of $\mathcal{Q}_{8}$ lying in $\mathrm{T}^{*}$ lies entirely in $G_{0}$ if $n \equiv 0,8(\bmod 12)$, and in $G_{2}$ if $n \equiv 2,6$ (mod 12). The complement of this copy of $\mathcal{Q}_{8}$ in $\mathrm{T}^{*}$ consists of elements of order 3 and 6 , and so lie in $G_{0}$ if $n \equiv 0(\bmod 6)$ and in $G_{2}$ if $n \equiv 2(\bmod 6)$ (thus the subgroups of $\mathrm{O}^{*}$ isomorphic to $\mathbb{Z}_{3}$ and $\mathbb{Z}_{6}$ lie in $G_{0}$ if $n \equiv 0(\bmod 6)$ and in $G_{2}$ if $n \equiv 2(\bmod 6))$. Thus $\mathrm{T}^{*}$ lies in $G_{0}$ if $n \equiv 0(\bmod 12)$, in $G_{2}$ if $n \equiv 2(\bmod 12)$, and lies in $G_{0} \cup G_{2}$ and meets both $G_{0}$ and $G_{2}$ if $n \equiv 6,8(\bmod 12)$.

In order to analyse the remaining possible subgroups $\mathcal{Q}_{16}, \mathrm{Dic}_{12}, \mathrm{Dic}_{20}$ of $\mathrm{O}^{*}$, as well as the other copy of $\mathcal{Q}_{8}$ lying in $\mathcal{Q}_{16}$, we must study the elements of $H \backslash \mathrm{T}^{*}$. They project to elements of $S_{4} \backslash A_{4}$, which are either 4-cycles, or transpositions. We analyse the geometric formulation of $\mathrm{O}^{*}$ described in Section 3 as being obtained from the action of $S_{4}$ on a cube, with the $n$ strings attached appropriately. The 4-cycles are realised by rotations by $\pi / 2$ about an axis which passes through the centres of two opposite faces. This gives rise to an element of $G_{0}$ if the $n$ marked points are not these central points (ie if $n \equiv 0,8(\bmod 12))$, and to elements of $G_{2}$ if some of the $n$ marked points are central points of the faces (ie if $n \equiv 2,6(\bmod 12))$. The transpositions are realised by rotations by $\pi$ about an axis which passes through the centres of two diagonally opposite edges. This gives rise to an element of $G_{0}$ if there are an even number of marked points on each edge (ie if $n \equiv 0,6,8,14(\bmod 24))$, and to elements of $G_{2}$ if there are an odd number of marked points on each edge (ie if $n \equiv 2,12,18,20(\bmod 24))$. Putting together these results with those for $\mathrm{T}^{*}$, if $H \cong \mathrm{O}^{*}$, we conclude that it lies in $G_{0}$ if $n \equiv 0(\bmod 24)$, in $G_{2}$ if $n \equiv 2(\bmod 24)$, and lies in $G_{0} \cup G_{2}$ and meets both $G_{0}$ and $G_{2}$ if $n \equiv 6,8,12,14,18,20(\bmod 24)$.

Now suppose that $H$ is a subgroup of a copy of $\mathrm{O}^{*}$ isomorphic to $\mathcal{Q}_{16}$. Such subgroups are the Sylow 2-subgroups of $\mathrm{O}^{*}$, so are conjugate. If $n \equiv 0(\bmod 24)($ resp. $n \equiv 2$ $(\bmod 24))$ then $\mathrm{O}^{*}$ lies in $G_{0}\left(\right.$ resp. $\left.G_{2}\right)$, and hence so does $\mathcal{Q}_{16}$. So suppose that 
$n \not \equiv 0,2(\bmod 24)$. Any subgroup of $\mathrm{O}^{*}$ isomorphic to $\mathcal{Q}_{16}$ contains elements of order 8 which lie in $\mathrm{O}^{*} \backslash \mathrm{T}^{*}$, and so are associated with the above 4-cycles. Further, $H$ projects to a subgroup of $S_{4}$ isomorphic to $\mathrm{D}_{8}$ which is generated by a 4-cycle and a transposition. Studying the associated rotations as above, if one has fixed points and the other not then automatically $H$ lies in $G_{0} \cup G_{2}$ and meets both $G_{0}$ and $G_{2}$. This occurs when $n \equiv 6,12,14,20(\bmod 24)$. So suppose that $n \equiv 8,18(\bmod 24)$.

If $n \equiv 8(\bmod 24)($ resp. $n \equiv 18(\bmod 24))$ then the elements of $H$ corresponding to the 4-cycles and the transpositions of $\mathrm{D}_{8}$ belong to $G_{0}$ (resp. $G_{2}$ ). Further, the remaining elements of $\mathrm{D}_{8}$ are products of such elements, and so the corresponding elements in $H$ are also elements of $\mathrm{T}^{*} \cong \mathcal{Q}_{8} \rtimes \mathbb{Z}_{3}$ of order 4 . But such elements lie in the $\mathcal{Q}_{8}$-factor. Since $n \equiv 8(\bmod 12)($ resp. $n \equiv 6(\bmod 12))$, this copy of $\mathcal{Q}_{8}$ lies in $G_{0}$ (resp. $G_{2}$ ), and hence so does the given subgroup $\mathcal{Q}_{16}$. Summing up, $H$ lies in $G_{0}$ if $n \equiv 0,8(\bmod 24)$, in $G_{2}$ if $n \equiv 2,18(\bmod 24)$, and lies in $G_{0} \cup G_{2}$ and meets both $G_{0}$ and $G_{2}$ if $n \equiv 6,12,14,20(\bmod 24)$.

Now suppose that $H$ is a subgroup of a copy of $\mathrm{O}^{*}$ isomorphic to $\mathrm{Dic}_{12}$. If $n \equiv 0$ $(\bmod 24)(\operatorname{resp} . n \equiv 2(\bmod 24))$ then $\mathrm{O}^{*}$ lies in $G_{0}\left(\right.$ resp. $\left.G_{2}\right)$, and hence so does $H$. So suppose that $n \neq \equiv 0,2(\bmod 24)$. Any subgroup of $\mathrm{O}^{*}$ isomorphic to $H$ projects onto a subgroup of $S_{4}$ isomorphic to $S_{3}$ which consists of 3-cycles and transpositions. Hence $H$ is generated by an element of order 4 lying in $\mathrm{O}^{*} \backslash \mathrm{T}^{*}$, and an element of order 6 , which lies in $\mathrm{T}^{*}$. The first element belongs to $G_{0}$ if $n \equiv 6,8,14$ $(\bmod 24)$ and to $G_{2}$ if $n \equiv 12,18,20(\bmod 24)$, while the second element belongs to $G_{0}$ if $n \equiv 6,12,18(\bmod 24)$ and to $G_{2}$ if $n \equiv 8,14,20(\bmod 24)$. Hence if $n \equiv 8,12,14,18 \equiv 24$ then $H$ lies in $G_{0} \cup G_{2}$ and meets both $G_{0}$ and $G_{2}$. The product of the two given generators is also of order 4 and so lies in $G_{0}$ if $n \equiv 6$ $(\bmod 24)$, and in $G_{2}$ if $n \equiv 20(\bmod 24)$. Thus $H$ lies in $G_{0}$ if $n \equiv 0,6(\bmod 24)$, in $G_{2}$ if $n \equiv 2,20(\bmod 24)$, and lies in $G_{0} \cup G_{2}$ and meets both $G_{0}$ and $G_{2}$ if $n \equiv 8,12,14,18(\bmod 24)$.

Now suppose that $H$ is a subgroup of a copy of $\mathrm{O}^{*}$ isomorphic to $\mathbb{Z}_{4}$. There are two possibilities. If it is contained in the copy of $\mathcal{Q}_{8}$ lying in the subgroup $\mathrm{T}^{*}$, from the results for $\mathcal{Q}_{8}$, we see that $H$ lies in $G_{0}$ if $n \equiv 0,8(\bmod 12)$, and in $G_{2}$ if $n \equiv 2,6(\bmod 12)$. The second possibility is that $H$ possesses elements in $\mathrm{O}^{*} \backslash \mathrm{T}^{*}$, and emanates from the rotation of order 2 whose permutation is a transposition. Thus it is contained in $G_{0}$ if $n \equiv 0,6,8,14(\bmod 24)$, and to $G_{2}$ if $n \equiv 2,12,18,20$ $(\bmod 24)$.

Finally, suppose that $H$ is a subgroup of a copy of $\mathrm{O}^{*}$ isomorphic to $\mathcal{Q}_{8}$. Again there are two possibilities. If $H$ lies in the subgroup $\mathrm{T}^{*}$, it is contained in $G_{0}$ if $n \equiv 0,8$ $(\bmod 12)$, and to $G_{2}$ if $n \equiv 2,6(\bmod 12)$. The second possibility is that it projects 
to a subgroup of $S_{4}$ generated by two transpositions having disjoint support. Such a subgroup thus has four elements of order 4 in $\mathrm{O}^{*} \backslash \mathrm{T}^{*}$ and two in $\mathrm{T}^{*}$. From the results obtained in the case of $\mathbb{Z}_{4}$, we see that $H$ lies in $G_{0}$ if $n \equiv 0,8(\bmod 24)$, in $G_{2}$ if $n \equiv 2,18(\bmod 24)$, and lies in $G_{0} \cup G_{2}$ and meets both $G_{0}$ and $G_{2}$ if $n \equiv 6,12,14,20(\bmod 24)$.

\section{Realisation of finite groups as subgroups of the lower cen- tral and derived series of $B_{n}\left(\mathbb{S}^{2}\right)$}

In this section, we consider the realisation of the finite subgroups of Theorem 1.3 as subgroups of elements of the lower central series $\left\{\Gamma_{i}\left(B_{n}\left(\mathbb{S}^{2}\right)\right)\right\}_{n \in \mathbb{N}}$ and of the derived series $\left\{\left(B_{n}\left(\mathbb{S}^{2}\right)\right)^{(i)}\right\}_{i \geq 0}$ of $B_{n}\left(\mathbb{S}^{2}\right)$. By [26], we already know that if $4 \mid n$ then $\Gamma_{2}\left(B_{n}\left(\mathbb{S}^{2}\right)\right)$ has a subgroup isomorphic to $\mathcal{Q}_{8}$. If $n \geq 4$ is even but not divisible by 4 , we may ask if the same result is true if $4 \nmid n$. We start by proving Theorem 1.6, which is the case of the dicyclic groups. We then complete the analysis of the other finite subgroups in Proposition 5.1.

Proof of Theorem 1.6 Suppose that $n$ is even. Let $N \in\{n-2, n\}$, set $N=2^{l} k$ where $l \in \mathbb{N}$ and $k$ is odd, and let $x=\alpha_{0}$ (resp. $x=\alpha_{0} \alpha_{2} \alpha_{0}^{-1}$ ) if $N=n$ (resp. $N=n-2)$.

(1) Since $B_{n}\left(\mathbb{S}^{2}\right)$ has a subgroup $\left\langle x, T_{n}\right\rangle$ isomorphic to $\operatorname{Dic}_{4 N}=\operatorname{Dic}_{2^{l+2} k}$, the statement is true for $j=0$. So suppose the result holds for some $j \in\{0,1, \ldots, l-1\}$. Then $B_{n}\left(\mathbb{S}^{2}\right)$ contains $2^{j}$ copies of $\operatorname{Dic}_{2^{l+2-j} k}$ of the form $\left\langle x^{2^{j}}, x^{i} T_{n}\right\rangle$, for $i=$ $0,1, \ldots, 2^{j}-1$. Hence $\left\langle x^{2^{j+1}}, x^{i} T_{n}\right\rangle$ is a subgroup of $\left\langle x^{2^{j}}, x^{i} T_{n}\right\rangle$ isomorphic to Dic $_{2^{l+1-j} k}$. But since

$$
\left(x^{\left(2^{j}+i\right)} T_{n}\right)^{2}=x^{\left(2^{j}+i\right)} T_{n} x^{\left(2^{j}+i\right)} T_{n}^{-1} T_{n}^{2}=\Delta_{n},
$$

and

$$
x^{\left(2^{j}+i\right)} T_{n} \cdot x^{2^{j+1}}\left(x^{\left(2^{j}+i\right)} T_{n}\right)^{-1}=x^{-2^{j+1}},
$$

it follows that $\left\langle x^{2^{j+1}}, x^{\left(2^{j}+i\right)} T_{n}\right\rangle$ is also a subgroup of $\left\langle x^{2^{j}}, x^{i} T_{n}\right\rangle$ isomorphic to $\operatorname{Dic}_{2^{l+1-j} k}$.

If $q$ is any divisor of $k$, then replacing $x$ by $x^{q}$ yields also $2^{j}$ copies $\left\langle x^{2^{j} q}, x^{i q} T_{n}\right\rangle$, $i=0,1, \ldots, 2^{j}-1$, of $\operatorname{Dic}_{2^{l+2-j} k / q}$ for $j \in\{0,1, \ldots, l\}$.

(2) If $j=0$, then the statement holds trivially. So suppose that $j \geq 1$. From part (1), $\left\langle x^{2^{j} q}, x^{i q} T_{n}\right\rangle$ and $\left\langle x^{2^{j} q}, x^{i^{\prime} q} T_{n}\right\rangle$ are subgroups of $B_{n}\left(\mathbb{S}^{2}\right)$ isomorphic 
to $\operatorname{Dic}_{2^{l+2-j}} k / q$. Under the abelianisation homomorphism $\xi: B_{n}\left(\mathbb{S}^{2}\right) \longrightarrow \mathbb{Z}_{2(n-1)}$, $\xi(x)=\overline{n-1}$, and

$$
\xi\left(T_{n}\right)=\xi\left(\left(\sigma_{1} \cdots \sigma_{n-1}\right) \cdots\left(\sigma_{1} \sigma_{2}\right) \sigma_{1}\right)=\overline{n(n-1) / 2}= \begin{cases}\overline{0} & \text { if } n / 2 \text { is even } \\ \overline{n-1} & \text { if } n / 2 \text { is odd }\end{cases}
$$

Since $j \geq 1, \xi\left(x^{2^{j} q}\right)=\overline{0}$. Furthermore,

$$
\xi\left(x^{i q} T_{n}\right)= \begin{cases}\overline{0} & \text { if } n / 2+i \text { is even } \\ \overline{n-1} & \text { if } n / 2+i \text { is odd }\end{cases}
$$

So $\left\langle x^{2^{j} q}, x^{i q} T_{n}\right\rangle \subset \Gamma_{2}\left(B_{n}\left(\mathbb{S}^{2}\right)\right)$ if and only if $n / 2+i$ is even. Thus if $i-i^{\prime}$ is odd, the subgroups $\left\langle x^{2^{j} q}, x^{i q} T_{n}\right\rangle$ and $\left\langle x^{2^{j} q}, x^{i^{\prime} q} T_{n}\right\rangle$ cannot be conjugate. But by Proposition 1.5(2), these are precisely the conjugacy classes of subgroups isomorphic to $\operatorname{Dic}_{2^{l+2-j}} k / q$. The result follows.

From this, we may deduce Proposition 1.7.

Proof of Proposition 1.7 We use the notation of the proof of Theorem 1.6. If $j=0$ and $q$ is an odd divisor of $n$ then there is just one conjugacy class of the abstract group $\operatorname{Dic}_{4 n / q}$, which is realised as $\left\langle x^{q}, T_{n}\right\rangle$. Now $x^{q} \notin \Gamma_{2}\left(B_{n}\left(\mathbb{S}^{2}\right)\right)$, so $\operatorname{Dic}_{4 n / q} \not \subset$ $\Gamma_{2}\left(B_{n}\left(\mathbb{S}^{2}\right)\right)$.

If $j \geq 1$ then as we saw in the proof of Theorem 1.6, $\left\langle x^{2^{j} q}, x^{i q} T_{n}\right\rangle \subset \Gamma_{2}\left(B_{n}\left(\mathbb{S}^{2}\right)\right)$ if and only if $n / 2+i$ is even. So with $i=0,1$, one of $\left\langle x^{2^{j} q}, T_{n}\right\rangle$ and $\left\langle x^{2^{j} q}, x^{q} T_{n}\right\rangle$ is contained in $\Gamma_{2}\left(B_{n}\left(\mathbb{S}^{2}\right)\right)$, while the other is not.

Finally, let $N$ be the element of $\{n, n-2\}$ divisible by 4 . Then $l \geq 2$, and taking $q=k$ and $j=l-1$, from the previous paragraph, one of $\left\langle x^{N / 2}, T_{n}\right\rangle$ and $\left\langle x^{N / 2}, x^{k} T_{n}\right\rangle$ (the two nonconjugate copies of $\left.\mathcal{Q}_{8}\right)$ belongs to $\Gamma_{2}\left(B_{n}\left(\mathbb{S}^{2}\right)\right)$, the other not.

We now give the analogous result for the cyclic and binary polyhedral subgroups of $B_{n}\left(\mathbb{S}^{2}\right)$.

Proposition 5.1 Let $G$ be a finite subgroup of $B_{n}\left(\mathbb{S}^{2}\right)$.

(1) Suppose that $G$ is cyclic.

(a) If $G$ is of order 2, then $G \subset \Gamma_{2}\left(B_{n}\left(\mathbb{S}^{2}\right)\right)$ if and only if $n$ is even.

(b) Suppose that $G$ is of order greater than or equal to 3. Then either:

(i) $|G|$ divides $2(n-1)$ in which case $G \not \subset \Gamma_{2}\left(B_{n}\left(\mathbb{S}^{2}\right)\right)$, or

(ii) $|G|$ divides $2(n-i)$, where $i \in\{0,2\}$. In this case, $G \subset \Gamma_{2}\left(B_{n}\left(\mathbb{S}^{2}\right)\right)$ if and only if $|G|$ divides $n-i$. 
(2) Suppose that $G$ is a subgroup of order at least 3 of some binary polyhedral subgroup $H$ of $B_{n}\left(\mathbb{S}^{2}\right)$.

(a) Suppose that $H \cong \mathrm{T}^{*}$ in the case that $\mathrm{T}^{*}$ is maximal. Then $G \subset \Gamma_{2}\left(B_{n}\left(\mathbb{S}^{2}\right)\right)$ if $G \cong \mathbb{Z}_{4}, \mathcal{Q}_{8}$, and $G \not \subset \Gamma_{2}\left(B_{n}\left(\mathbb{S}^{2}\right)\right)$ if $G \cong \mathbb{Z}_{3}, \mathbb{Z}_{6}, \mathrm{~T}^{*}$.

(b) Suppose that $H \cong \mathrm{I}^{*}$ in the case that $\mathrm{I}^{*}$ is maximal. Then $G \subset \Gamma_{2}\left(B_{n}\left(\mathbb{S}^{2}\right)\right)$.

(c) Suppose that $H \cong \mathrm{O}^{*}$ in the case that $\mathrm{O}^{*}$ is maximal. If $G$ is contained in the subgroup $K$ of $H$ isomorphic to $\mathrm{T}^{*}$ then $G \subset \Gamma_{2}\left(B_{n}\left(\mathbb{S}^{2}\right)\right)$. If $G \not \subset K$ then $G \subset \Gamma_{2}\left(B_{n}\left(\mathbb{S}^{2}\right)\right)$ if $n \equiv 0,2,8,18(\bmod 24)$, and $G \not \subset \Gamma_{2}\left(B_{n}\left(\mathbb{S}^{2}\right)\right)$ if $n \equiv 6,12,14,20(\bmod 24)$.

Proof We set $\Gamma_{2}=\Gamma_{2}\left(B_{n}\left(\mathbb{S}^{2}\right)\right)$. If $G$ is of order 2 , then $G=\left\langle\Delta_{n}\right\rangle$ and as $\xi\left(\Delta_{n}\right)=$ $\overline{n(n-1)}$, it follows easily that $G \subset \Gamma_{2}$ if and only if $n$ is even. We assume from now on that $|G| \geq 3$. Since $\Gamma_{2}$ is normal in $B_{n}\left(\mathbb{S}^{2}\right)$, we may work up to conjugation.

First suppose that $G$ is cyclic. Then by Theorem 1.1, it is conjugate to a subgroup of $\left\langle\alpha_{i}\right\rangle$ for some $i \in\{0,1,2\}$. If $i=1$ then $\xi\left(\alpha_{1}^{j}\right)=\overline{j n}$ for all $j \in \mathbb{Z}$. If $\alpha_{1}^{j} \in \Gamma_{2}$ then there exists $k \in \mathbb{Z}$ such that $j n=2 k(n-1)$, thus $(n-1) \mid j$, and so $j=l(n-1)$ for some $l \in \mathbb{Z}$. But then $\alpha_{1}^{j}=\alpha_{1}^{l(n-1)} \in\left\langle\Delta_{n}\right\rangle$. We conclude that $\left\langle\alpha_{1}\right\rangle \cap \Gamma_{2} \subset\left\langle\Delta_{n}\right\rangle$. Hence $G \not \subset \Gamma_{2}$.

Suppose then that $G$ is conjugate to a subgroup of $\left\langle\alpha_{i}\right\rangle$, where $i=0,2$. Set $k=|G|$. Then $\xi\left(\alpha_{i}\right)=\overline{n-1}, k \mid 2(n-i)$, and up to conjugacy, $G=\left\langle\alpha_{i}^{2(n-i) / k}\right\rangle$. So $G \subset \Gamma_{2}$ if and only if $2(n-i) / k$ is even, which is equivalent to $k \mid n-i$. Thus if $G$ is conjugate to a subgroup of $\left\langle\alpha_{i}\right\rangle$, where $i=0,2$, we have:

$$
G \subset \Gamma_{2} \Longleftrightarrow|G| \mid(n-i) .
$$

Now suppose that $H$ is isomorphic to $\mathrm{T}^{*}$ in the case that $\mathrm{T}^{*}$ is maximal, so that $n \equiv 4(\bmod 6)$. If $G$ is isomorphic to $\mathrm{T}^{*}, \mathbb{Z}_{6}$ or $\mathbb{Z}_{3}$ then the order 3 elements lie in $G_{1} \backslash\left\langle\Delta_{n}\right\rangle$, and from the cyclic case, it follows that $G \not \subset \Gamma_{2}$. So assume that $G$ is isomorphic to either $\mathbb{Z}_{4}$ or $\mathcal{Q}_{8}$. Since $\mathcal{Q}_{8}$ is generated by elements of order 4 , it suffices to analyse the case $\mathbb{Z}_{4}$. By Proposition 4.1, $G$ lies in $G_{0}$ if $n \equiv 4(\bmod 12)$, and in $G_{2}$ if $n \equiv 10(\bmod 12)$. In both cases, $G \subset \Gamma_{2}$ by Equation (5-1).

Now suppose that $H$ is isomorphic to $\mathrm{I}^{*}$ in the case that $\mathrm{I}^{*}$ is maximal, so that $n \equiv 0,2,12,20(\bmod 30)$. We claim that $G \subset \Gamma_{2}$ whatever the value of $n$. To see this, it suffices to check that all of the maximal cyclic subgroups $\mathbb{Z}_{4}, \mathbb{Z}_{6}, \mathbb{Z}_{10}$ of I* are contained in $\Gamma_{2}$. This follows easily from Proposition 4.1 and Equation (5-1).

Now suppose that $H$ is isomorphic to $\mathrm{O}^{*}$ in the case that $\mathrm{O}^{*}$ is maximal, so $n \equiv 0,2$ ( $\bmod 6)$. Again it suffices to consider the maximal cyclic subgroups $\mathbb{Z}_{4}, \mathbb{Z}_{6}$ and $\mathbb{Z}_{8}$ of $\mathrm{O}^{*}$. Applying Proposition 4.1 and Equation (5-1), we obtain the following results: 
(1) If $G$ is isomorphic to $\mathbb{Z}_{8}$, it projects to a subgroup of $S_{4}$ generated by a 4-cycle. Then $G \subset G_{0}$ if $n \equiv 0,8(\bmod 12)$, and $G \subset G_{2}$ if $n \equiv 2,6(\bmod 12)$, and so $G \subset \Gamma_{2}$ if $n \equiv 0,2,8,18(\bmod 24)$, and $G \not \subset \Gamma_{2}$ if $n \equiv 6,12,14,20(\bmod 24)$.

(2) If $G$ is isomorphic to $\mathbb{Z}_{6}$ then $G \subset \Gamma_{2}$.

(3) If $G$ is isomorphic to $\mathbb{Z}_{4}$, there are two possibilities. If $G$ lies in the subgroup $K$ of $\mathrm{O}^{*}$ isomorphic to $\mathrm{T}^{*}$ then $G \subset \Gamma_{2}$. Otherwise $G$ is generated by an element of order 4 not belonging to $K$, in which case we obtain the same answer as for $\mathbb{Z}_{8}$.

Since every cyclic subgroup of order 3 of $\mathrm{O}^{*}$ is contained in one of order 6 , this gives the results if $G$ is cyclic. Suppose now that $G=K$. Then $G$ is generated by the elements of order 6 and the elements of order 4 belonging to $K$, so $G \subset \Gamma_{2}$.

If $G$ is abstractly isomorphic to $\mathcal{Q}_{16}$ then it is generated by elements of order 8, elements of order 4 lying in $K$, and elements of order 4 not lying in $K$. From above, we have that $G \subset \Gamma_{2}$ if $n \equiv 0,2,8,18(\bmod 24)$, and $G \not \subset \Gamma_{2}$ if $n \equiv 6,12,14,20$ $(\bmod 24)$.

If $G$ is abstractly isomorphic to $\mathcal{Q}_{8}$ then there are two possibilities: either $G$ lies in $K$, so is contained in $\Gamma_{2}$, or else it is generated by elements of order 4 not belonging to $K$. In this case, from above, $G \subset \Gamma_{2}$ if $n \equiv 0,2,8,18(\bmod 24)$, and $G \not \subset \Gamma_{2}$ if $n \equiv 6,12,14,20(\bmod 24)$.

Finally, suppose that $G$ is abstractly isomorphic to $\operatorname{Dic}_{12}$. Then it projects to a copy of $S_{3}$ in $S_{4}$. From above, it follows that $G \subset \Gamma_{2}$ if $n \equiv 0,2,8,18(\bmod 24)$, and $G \not \subset \Gamma_{2}$ if $n \equiv 6,12,14,20(\bmod 24)$.

Remark 5.2 Having dealt with the behaviour of the finite subgroups relative to the commutator subgroup of $B_{n}\left(\mathbb{S}^{2}\right)$, one might ask what happens for the higher elements of the lower central series $\left\{\Gamma_{i}\left(B_{n}\left(\mathbb{S}^{2}\right)\right)\right\}_{n \in \mathbb{N}}$ and of the derived series $\left\{\left(B_{n}\left(\mathbb{S}^{2}\right)\right)^{(i)}\right\}_{i \geq 0}$ of $B_{n}\left(\mathbb{S}^{2}\right)$. But if $n \neq 2$ (resp. $n \geq 5$ ), the lower central series (resp. derived series) of $B_{n}\left(\mathbb{S}^{2}\right)$ is stationary from the commutator subgroup onwards [23]. It just remains to look at the derived series of $B_{4}\left(\mathbb{S}^{2}\right)$. Recall from that paper that $\left(B_{4}\left(\mathbb{S}^{2}\right)\right)^{(1)}$ is a semi-direct product of $\mathcal{Q}_{8}$ by a free group $\mathbb{F}_{2}$ of rank two, that $\left(B_{4}\left(\mathbb{S}^{2}\right)\right)^{(2)}$ is a semi-direct product of $\mathcal{Q}_{8}$ by the derived subgroup $\left(\mathbb{F}_{2}\right)^{(1)}$ of $\mathbb{F}_{2}$, that $\left(B_{4}\left(\mathbb{S}^{2}\right)\right)^{(3)}$ is the direct product of $\left\langle\Delta_{4}\right\rangle$ by $\left(\mathbb{F}_{2}\right)^{(2)}$, and that $\left(B_{4}\left(\mathbb{S}^{2}\right)\right)^{(i+1)} \cong\left(\mathbb{F}_{2}\right)^{(i)}$ for all $i \geq 3$. Thus there is a copy of $\mathcal{Q}_{8}$ which lies in $\left(B_{4}\left(\mathbb{S}^{2}\right)\right)^{(2)}$ but not in $\left(B_{4}\left(\mathbb{S}^{2}\right)\right)^{(3)}$. The full twist remains until $\left(B_{4}\left(\mathbb{S}^{2}\right)\right)^{(3)}$, and then $\left(B_{4}\left(\mathbb{S}^{2}\right)\right)^{(4)}$ is torsion free. 


\section{A proof of Murasugi's theorem}

Let $H_{1}, H_{2}$ be isomorphic finite cyclic subgroups of $\mathcal{M}_{0, n}$. From Theorem 2.2, if $n$ is odd, or if $n$ is even and $\left|H_{1}\right|=\left|H_{2}\right| \neq 2$ then $H_{1}$ and $H_{2}$ are conjugate. If $n$ is even, there are exactly two conjugacy classes of subgroups of $\mathcal{M}_{0, n}$ of order 2 , and thus there are exactly two conjugacy classes of subgroups of $B_{n}\left(\mathbb{S}^{2}\right)$ of order 4 .

The next proposition follows from Section 2.

Proposition 6.1 Let $G_{1}, G_{2}$ be isomorphic finite cyclic subgroups of order $m$ of $B_{n}\left(\mathbb{S}^{2}\right)$. If $n$ is odd, or if $n$ is even and $m \neq 4$ then $G_{1}$ and $G_{2}$ are conjugate. If $n$ is even, there are exactly two conjugacy classes of subgroups of $B_{n}\left(\mathbb{S}^{2}\right)$ of order 4 .

If $n$ is even then $\alpha_{0}^{n / 2}$ and $\alpha_{2}^{(n-2) / 2}$ are of order 4, and they generate nonconjugate subgroups since their images in $S_{n}$ are not conjugate, which yields the two conjugacy classes of $\mathbb{Z}_{4}$ of Proposition 6.1. From this, we may deduce Theorem 1.1.

Proof of Theorem 1.1 Let $x \in B_{n}\left(\mathbb{S}^{2}\right)$ be a torsion element. Then $\langle x\rangle$ is contained in a maximal cyclic subgroup $C$ of one of the maximal finite subgroups $G$ of $B_{n}\left(\mathbb{S}^{2}\right)$ given by Theorem 1.3.

First suppose that $n$ is odd. Then $G$ is one of $\mathbb{Z}_{2(n-1)}, \operatorname{Dic}_{4 n}$ and $\operatorname{Dic}_{4(n-2)}$, and so $C$ must be one of $\mathbb{Z}_{2(n-1)}, \mathbb{Z}_{2 n}, \mathbb{Z}_{2(n-2)}$ and $\mathbb{Z}_{4}$. Hence $C$ is isomorphic to $\left\langle\alpha_{1}\right\rangle$, $\left\langle\alpha_{0}\right\rangle,\left\langle\alpha_{2}\right\rangle$ and $\left\langle\alpha_{1}^{(n-1) / 2}\right\rangle$ respectively. So by Proposition 6.1, $x$ is conjugate to a power of one of $\alpha_{0}, \alpha_{1}$ and $\alpha_{2}$ which proves the theorem in this case.

Now suppose that $n$ is even. If $C \cong \mathbb{Z}_{4}$ then $C$ is conjugate to one of $\left\langle\alpha_{0}^{n / 2}\right\rangle$ or $\left\langle\alpha_{2}^{(n-2) / 2}\right\rangle$, and the result holds. So suppose that $C \not \mathbb{Z}_{4}$. If $G$ is one of $\mathbb{Z}_{2(n-1)}$, $\operatorname{Dic}_{4 n}$ and $\operatorname{Dic}_{4(n-2)}$, then $C$ is one of $\mathbb{Z}_{2(n-1)}, \mathbb{Z}_{2 n}, \mathbb{Z}_{2(n-2)}$, and so is isomorphic to $\left\langle\alpha_{1}\right\rangle,\left\langle\alpha_{0}\right\rangle$, and $\left\langle\alpha_{2}\right\rangle$ respectively. If $G=\mathrm{T}^{*}(\operatorname{so} n \equiv 4(\bmod 6))$ then $C \cong \mathbb{Z}_{6}$, and so is conjugate to $\left\langle\alpha_{1}^{(n-1) / 3}\right\rangle$. If $G=\mathrm{O}^{*}($ so $n \equiv 0,2(\bmod 6))$ then $C \cong \mathbb{Z}_{6}$ or $C \cong \mathbb{Z}_{8}$, and so is conjugate to $\left\langle\alpha_{0}^{n / 3}\right\rangle$ or $\left\langle\alpha_{2}^{(n-2) / 3}\right\rangle$. Finally, if $G=\mathrm{I}^{*}$ (so $n \equiv 0,2,12,20(\bmod 30))$ then $C$ is isomorphic to one of $\mathbb{Z}_{6}$ or $\mathbb{Z}_{10}$. If $C \cong \mathbb{Z}_{6}$ then $C$ is conjugate to $\left\langle\alpha_{0}^{n / 3}\right\rangle$ if $n \equiv 0,12(\bmod 30)$ or to $\left\langle\alpha_{2}^{(n-2) / 3}\right\rangle$ if $n \equiv 2,20$ $(\bmod 30)$. If $C \cong \mathbb{Z}_{10}$ then $C$ is conjugate to $\left\langle\alpha_{0}^{n / 5}\right\rangle$ if $n \equiv 0,20(\bmod 30)$ or to $\left\langle\alpha_{2}^{(n-2) / 5}\right\rangle$ if $n \equiv 2,12(\bmod 30)$. In all cases, $x$ is conjugate to a power of one of $\alpha_{0}, \alpha_{1}$ and $\alpha_{2}$, which completes the proof of the theorem.

\section{References}

[1] A Adem, R J Milgram, Cohomology of finite groups, Grundlehren series 309, Springer, Berlin (1994) MR1317096 
[2] E Artin, Theorie der Zöpfe, Abhandlungen Hamburg 4 (1925) 47-72

[3] E Artin, Theory of braids, Ann. of Math. (2) 48 (1947) 101-126 MR0019087

[4] D J Benson, F R Cohen, Mapping class groups of low genus and their cohomology, Mem. Amer. Math. Soc. 90 (1991) iv+104 MR1052554

[5] J S Birman, Braids, links, and mapping class groups, Annals of Math. Studies 82, Princeton University Press (1974) MR0375281

[6] KS Brown, Cohomology of groups, Graduate Texts in Mathematics 87, Springer, New York (1982) MR672956

[7] $\mathbf{R}$ Brown, Algebraic topology discussion list (2004) Online at http://www . lehigh. edu/ dmd1/pz119.txt

[8] F R Cohen, On the mapping class groups for punctured spheres, the hyperelliptic mapping class groups, SO(3), and $\operatorname{Spin}^{c}(3)$, Amer. J. Math. 115 (1993) 389-434 MR1216436

[9] F R Cohen, S Gitler, On loop spaces of configuration spaces, Trans. Amer. Math. Soc. 354 (2002) 1705-1748 MR1881013

[10] HS M Coxeter, Regular complex polytopes, second edition, Cambridge University Press, Cambridge (1991) MR1119304

[11] HS M Coxeter, W OJ Moser, Generators and relations for discrete groups, fourth edition, Ergebnisse series 14, Springer, Berlin (1980) MR562913

[12] C J Earle, J Eells, The diffeomorphism group of a compact Riemann surface, Bull. Amer. Math. Soc. 73 (1967) 557-559 MR0212840

[13] E Fadell, Homotopy groups of configuration spaces and the string problem of Dirac, Duke Math. J. 29 (1962) 231-242 MR0141127

[14] E R Fadell, S Y Husseini, Geometry and topology of configuration spaces, Springer Monographs in Math., Springer, Berlin (2001) MR1802644

[15] E Fadell, J Van Buskirk, The braid groups of $E^{2}$ and $S^{2}$, Duke Math. J. 29 (1962) 243-257 MR0141128

[16] R Fox, L Neuwirth, The braid groups, Math. Scand. 10 (1962) 119-126 MR0150755

[17] R Gillette, J Van Buskirk, The word problem and consequences for the braid groups and mapping class groups of the 2-sphere, Trans. Amer. Math. Soc. 131 (1968) 277-296 MR0231894

[18] M Golasiński, D L Gonçalves, Spherical space forms-homotopy types and selfequivalences, from: "Categorical decomposition techniques in algebraic topology (Isle of Skye, 2001)”, Progr. Math. 215, Birkhäuser, Basel (2004) 153-165 MR2039764

[19] M Golasiński, D L Gonçalves, Spherical space forms-homotopy types and selfequivalences for the groups $\mathbb{Z} / a \rtimes \mathbb{Z} / b$ and $\mathbb{Z} / a \rtimes\left(\mathbb{Z} / b \times \mathbb{Q}_{2}{ }^{i}\right)$, Topology Appl. 146/147 (2005) 451-470 MR2107164 
[20] M Golasiński, D L Gonçalves, Homotopy types of orbit spaces and their selfequivalences for the periodic groups $\mathbb{Z} / a \rtimes\left(\mathbb{Z} / b \times T_{n}^{*}\right)$ and $\mathbb{Z} / a \rtimes\left(\mathbb{Z} / b \times O_{n}^{*}\right)$, J. Homotopy Relat. Struct. 1 (2006) 29-45 MR2264736

[21] M Golasiński, D L Gonçalves, Spherical space forms: homotopy types and selfequivalences for the group $(\mathbb{Z} / a \rtimes \mathbb{Z} / b) \times \mathrm{SL}_{2}\left(\mathbb{F}_{p}\right)$, Canad. Math. Bull. 50 (2007) 206-214 MR2317443

[22] M Golasiński, D L Gonçalves, Spherical space forms - homotopy types and selfequivalences for the group $(\mathbb{Z} / a \rtimes \mathbb{Z} / b) \times \mathrm{TL}_{2}\left(\mathbb{F}_{p}\right)$, preprint (2007)

[23] D L Gonçalves, J Guaschi, The lower central and derived series of the braid groups of the sphere, to appear in Trans. of Amer. Math. Soc. arXiv:math/0603701

[24] D L Gonçalves, J Guaschi, The roots of the full twist for surface braid groups, Math. Proc. Cambridge Philos. Soc. 137 (2004) 307-320 MR2092062

[25] D L Gonçalves, J Guaschi, The braid group $B_{n, m}\left(\mathbb{S}^{2}\right)$ and a generalisation of the Fadell-Neuwirth short exact sequence, J. Knot Theory Ramifications 14 (2005) 375-403 MR2149513

[26] D L Gonçalves, J Guaschi, The quaternion group as a subgroup of the sphere braid groups, Bull. Lond. Math. Soc. 39 (2007) 232-234 MR2323453

[27] M-E Hamstrom, Homotopy groups of the space of homeomorphisms on a 2-manifold, Illinois J. Math. 10 (1966) 563-573 MR0202140

[28] V L Hansen, Braids and coverings: selected topics, London Math. Soc. Student Texts 18, Cambridge University Press (1989) MR1247697 With appendices by Lars Gæde and Hugh R Morton

[29] D L Johnson, Presentations of groups, London Math. Soc. Lecture Notes Series 22, Cambridge University Press (1976) MR0396763

[30] SP Kerckhoff, The Nielsen realization problem, Bull. Amer. Math. Soc. (N.S.) 2 (1980) 452-454 MR561531

[31] V Lin, Configuration spaces of $\mathbb{C}$ and $\mathbb{C P}^{1}$ : some analytic properties arXiv: math. AG/0403120

[32] W Magnus, Über Automorphismen von Fundamentalgruppen berandeter Flächen, Math. Ann. 109 (1934) 617-646 MR1512913

[33] W Magnus, A Karrass, D Solitar, Combinatorial group theory, revised edition, Dover Publications, New York (1976) MR0422434 Presentations of groups in terms of generators and relations

[34] K Murasugi, Seifert fibre spaces and braid groups, Proc. London Math. Soc. (3) 44 (1982) 71-84 MR642793

[35] MHA Newman, On a string problem of Dirac, J. London Math. Soc. 17 (1942) 173-177 MR0008156 
[36] G P Scott, The space of homeomorphisms of a 2-manifold, Topology 9 (1970) 97-109 MR0264676

[37] M Stukow, Conjugacy classes of finite subgroups of certain mapping class groups, Turkish J. Math. 28 (2004) 101-110 MR2062555

[38] C B Thomas, Elliptic structures on 3-manifolds, Lecture Notes in Math., U. of Chicago Department of Math., Chicago, IL (1983) MR749388

[39] J G Thompson, Note on H(4), Comm. Algebra 22 (1994) 5683-5687 MR1298742

[40] J Van Buskirk, Braid groups of compact 2-manifolds with elements of finite order, Trans. Amer. Math. Soc. 122 (1966) 81-97 MR0189013

[41] O Zariski, The topological discriminant group of a Riemann surface of genus $p$, Amer. J. Math. 59 (1937) 335-358 MR1507244

Departamento de Matemática - IME-USP

Caixa Postal 66281 - Ag. Cidade de São Paulo, CEP: 05314-970 - São Paulo - SP, Brazil

Laboratoire de Mathématiques Nicolas Oresme UMR CNRS 6139, Université de Caen

BP 5186, 14032 Caen Cedex, France

dlgoncal@ime.usp.br, guaschi@math.unicaen.fr

Received: 26 November 2007 Revised: 11 February 2008 